\title{
The Random Multisection Problem, Travelling Waves, and the Distribution of the Height of $m$-Ary Search Trees
}

\author{
Brigitte Chauvin and Michael Drmota
}

November 25, 2005

\begin{abstract}
The purpose of this article is to show that the distribution of the longest fragment in the random multisection problem after $k$ steps and the height of $m$-ary search trees (and some extensions) are not only closely related in a formal way but both can be asymptotically described with the same distribution function that has to be shifted in a proper way (travelling wave).

The crucial property for the proof is a so-called intersection property that transfers inequalities between two distribution functions (resp. of their Laplace transforms) from one level to the next. It is conjectured that such intersection properties hold in a much more general context. If this property is verified convergence to a travelling wave follows almost automatically.
\end{abstract}

\section{Introduction}

In this paper we study concentration properties of several extremal parameters, the shortest and longest fragment in a fragmentation process, the leftmost and rightmost particle in a branching random walk and the height and saturation level in some random trees (see Theorems 2.1-2.3). For all these parameters we observe the same phenomenon, the asymptotic distribution can be described with the help of a travelling wave, that is, the distribution centered at the median remains almost constant and consequently the distribution is concentrated around its median. ${ }^{1}$

In fact, we will concentrate on special cases that have nice analytic properties. However, the core of the proof is an intersection property (Lemma 4.3 for the multisection problem, Lemma 4.12 for $m$-ary search trees). In particular, in the last section (Section 5 ) we show in the framework of branching random walks that an intersection property always implies convergence to a travelling wave and, thus, concentration around the median (see Theorem 5.1).

\footnotetext{
${ }^{1}$ The interpretation of the result for the height and saturation level of trees is a little bit different (compare with Theorem 2.3) but of similar flavour.
} 


\section{Results}

In this section we state our results. We start with Theorem 2.1 on the random multisection problem, reformulate it in terms of branching random walks (Theorem 2.2) and close this section with Theorem 2.3 on the height and saturation level of $m$-ary search trees. Theorem 2.3 is probably the most interesting result. However, since the discussion of this result makes use of branching random walks and also of the multisection problem the other two theorems are stated first.

The Random Bisection Problem or Random Fragmentation Problem (see [3, 18, 19]) is defined as follows. An interval of length $x$ is cut into two halves of length $x_{1}=V x$ and $x_{2}=$ $(1-V) x$, where $V$ is a random variable taking values on $[0,1]$. Next, each of these two fragments is cut again into two parts (independently of the other and previous cuts). After the $k$-th step, there are $2^{k}$ fragments whose lengths are correlated random variables. Given the initial length $x$, the problem is to determine the probability $P_{k}(x, l)$ that each of the $2^{k}$ fragments is shorter or equal than $l$, that is, the distribution function of the longest fragment. Obviously this problem is homogeneous in $x$ and $l$. This motivates us to define $\bar{P}_{k}(x)=P_{k}(x, 1)$, that is, $P_{k}(x, l)=\bar{P}_{k}(x / l)$. By definition we have $\bar{P}_{0}(x)=1$ for $0 \leq x<1, \bar{P}_{0}(x)=0$ for $x \geq 1$, and recursively

$$
\bar{P}_{k+1}(x)=\mathbf{E}\left(\bar{P}_{k}(x V) \bar{P}_{k}(x(1-V))\right) .
$$

Similarly we can also consider the probability $Q_{k}(x, l)$ that each of the $2^{k}$ fragments is longer than $l$, that is, 1 minus the distribution function of the shortest fragment. With $\bar{Q}_{k}(x)=Q_{k}(x, 1)$ we obtain as above $\bar{Q}_{0}(x)=0$ for $0 \leq x<1, \bar{Q}_{0}(x)=1$ for $x \geq 1$, and recursively

$$
\bar{Q}_{k+1}(x)=\mathbf{E}\left(\bar{Q}_{k}(x V) \bar{Q}_{k}(x(1-V))\right) .
$$

In the same way we can define a Multisection Problem. Let $m \geq 2$ and $V_{1}, \ldots, V_{m}$ be random variables taking values on $[0,1]$ with $V_{1}+\cdots+V_{m}=1$. An interval of length $x$ is partitioned into $m$ parts of lengths $x V_{1}, x V_{2}, \ldots, x V_{m}$. Now the recurrence for $\bar{P}_{k}(x)$ is given by

$$
\bar{P}_{k+1}(x)=\mathbf{E}\left(\bar{P}_{k}\left(x V_{1}\right) \cdots \bar{P}_{k}\left(x V_{m}\right)\right) .
$$

In what follows we will always assume that $\left(V_{1}, \ldots, V_{m}\right)$ is (what we call) $t$-beta-distributed, where $t$ is a non-negative integer parameter. This means that

$$
\begin{aligned}
& \mathbf{E} f\left(V_{1}, \ldots, V_{m}\right)=\frac{(m(t+1)-1) !}{(t !)^{m}} \times \\
& \int_{0}^{1} \int_{0}^{1-x_{1}} \cdots \int_{0}^{1-x_{1}-\cdots-x_{m-2}}\left(x_{1} x_{2} \cdots x_{m-1}\left(1-x_{1}-\cdots-x_{m-1}\right)\right)^{t} \times \\
& \quad f\left(x_{1}, \ldots, x_{m-1}, 1-x_{1}-\cdots-x_{m-1}\right) d x_{m-1} d x_{m-2} \cdots d x_{1} .
\end{aligned}
$$

In other words, the random vector $\left(V_{1}, \ldots, V_{m}\right)$ is concentrated on the set $\left\{\left(x_{1}, x_{2}, \ldots, x_{m}\right) \in[0,1]^{m}: x_{1}+x_{2}+\cdots+x_{m}=1\right\}$ and the density of the distribution is 
given by

$$
\frac{(m(t+1)-1) !}{(t !)^{m}}\left(x_{1} x_{2} \cdots x_{m}\right)^{t} .
$$

We remark that, for large $t$, the distribution is approximated by the point distribution that is concentrated at $x_{1}=x_{2}=\cdots=x_{m}=\frac{1}{m}$. The fragments in the multisection problem will be more balanced for large $t$.

We will see in the sequel that these kinds of distributions occur naturally in the context of socalled fringe balanced $m$-ary search trees. Furthermore, relations of the form (1) can be restated as convolution identities and, thus, lead to a differential equation for the Laplace transform of $F$ that can be tackled with the help of our methods. This was a strong motivation for us to restrict ourselves to $t$-beta distributions. Of course, this is only a very special case of a fragmentation process (see [3]). We cannot contribute much to the general situation, see section 5 . Nevertheless we can provide a complete answer to the above special case as it was predicted by [18].

Theorem 2.1 Let $m \geq 2$ and $t \geq 0$ be integers and $\left(V_{1}, \ldots, V_{m}\right)$ be $t$-beta-distributed. Suppose that $\bar{P}_{k}(x)$ and $\bar{Q}_{k}(x)$ are the distribution functions of the corresponding multisection problem. Then there exist continuous functions $F(x)$ and $G(x)$ such that

$$
\bar{P}_{k}(x)=F\left(x / x_{k}\right)+o(1) \quad \text { and } \quad \bar{Q}_{k}(x)=G\left(x / y_{k}\right)+o(1)
$$

uniformly for $x \geq 0$ as $k \rightarrow \infty$, where $x_{k}$ and $y_{k}$ are defined by $\bar{P}_{k}\left(x_{k}\right)=\bar{Q}_{k}\left(y_{k}\right)=1 / 2$. They are asymptotically given by

$$
\log x_{k}=k \log \rho_{1}+\Theta(\log k) \quad \text { and } \quad \log y_{k}=k \log \rho_{2}+\Theta(\log k),
$$

where $\rho_{1}, \rho_{2}$ are defined as in Lemma 3.2.

Furthermore, there exist constants $C_{1}, C_{2}>0, \gamma_{1}, \gamma_{2}>1$, and $\beta_{1}, \beta_{2}>0$ with

$$
\begin{aligned}
\bar{P}_{k}(x) & =O\left(e^{-C_{1}\left(x / x_{k}\right)^{\gamma_{1}}}\right) \quad\left(\text { for } x \geq x_{k}\right), \\
1-\bar{P}_{k}(x) & =O\left(\left(x / x_{k}\right)^{\beta_{1}}\right) \quad\left(\text { for } x \leq x_{k}\right), \\
\bar{Q}_{k}(x) & =O\left(e^{-C_{2}\left(x / y_{k}\right)^{-\gamma_{2}}}\right) \quad\left(\text { for } x \leq y_{k}\right), \\
1-\bar{Q}_{k}(x) & =O\left(\left(x / y_{k}\right)^{-\beta_{2}}\right) \quad\left(\text { for } x \geq y_{k}\right) .
\end{aligned}
$$

A branching random walk is a sequence of point processes $Z_{k}$, where $Z_{0}=\delta_{0}$ and $Z_{k+1}$ evolves from $Z_{k}$ by splitting each particle of $Z_{k}$ (independently of one another) into a random number $N$ of points with displacements determined by a given point process $Z=\delta_{X_{1}}+\cdots+\delta_{X_{N}}{ }^{2}$ For example, if $N \equiv m$ and $X_{1}=\log \left(1 / V_{1}\right), \ldots, X_{m}=\log \left(1 / V_{m}\right)$ with $V_{1} \ldots, V_{m}$ from above

\footnotetext{
${ }^{2} \delta_{x}$ denotes the probability measure concentrated at the point $x$.
} 
then the distribution of $L_{k}$, the left most particle at stage $k$, (and of $R_{k}$, the corresponding right most particle) are given by

$$
\operatorname{Pr}\left\{L_{k}>x\right\}=\bar{P}_{k}\left(e^{x}\right) \text { and } \operatorname{Pr}\left\{R_{k} \leq x\right\}=\bar{Q}_{k}\left(e^{x}\right) .
$$

Thus, we can reformulate Theorem 2.1 in terms of the distribution of $L_{k}$ and $R_{k}$ as follows.

Theorem 2.2 Let $m \geq 2$ and $t \geq 0$ be integers and $\left(V_{1}, \ldots, V_{m}\right)$ be $t$-beta-distributed. Consider the branching random walk $Z_{k}$ with reproduction measure

$$
Z=\delta_{X_{1}}+\cdots+\delta_{X_{m}}
$$

where $X_{j}=\log \left(1 / V_{j}\right)(1 \leq j \leq m)$, and let $L_{k}$ resp. $R_{k}$ be the position of the leftmost resp. rightmost particle at level $k$. Then there exist functions $w_{1}(x)$ and $w_{2}(x)$ such that

$$
\operatorname{Pr}\left\{L_{k}>x\right\}=w_{1}\left(x-m_{1}(k)\right)+o(1) \quad \text { and } \quad \operatorname{Pr}\left\{R_{k} \leq x\right\}=w_{2}\left(x-m_{2}(k)\right)+o(1),
$$

where the medians $m_{1}(k)$ and $m_{2}(k)$ are defined by $\operatorname{Pr}\left\{L_{k} \leq m_{1}(k)\right\}=\operatorname{Pr}\left\{R_{k} \leq m_{2}(k)\right\}=1 / 2$ and are also given by $m_{1}(k)=\log x_{k}$ and $m_{2}(k)=\log y_{k}$ (with $x_{k}, y_{k}$ from Theorem 2.1). They are asymptotically given by

$$
m_{1}(k)=k \log \rho_{1}+\Theta(\log k) \quad \text { and } \quad m_{2}(k)=k \log \rho_{2}+\Theta(\log k) \quad(k \rightarrow \infty),
$$

where $\rho_{1}, \rho_{2}$ are defined as in Lemma 3.2.

Furthermore, there exist $C>0$ and $\eta>0$ with

$$
\operatorname{Pr}\left\{\left|L_{k}-m_{1}(k)\right|>x\right\} \leq C e^{-\eta x} \quad \text { and } \quad \operatorname{Pr}\left\{\left|R_{k}-m_{2}(k)\right|>x\right\} \leq C e^{-\eta x} .
$$

In particular we have, as $k \rightarrow \infty$,

$$
\operatorname{Var} L_{k}=O(1) \quad \text { and } \quad \operatorname{Var} R_{k}=O(1)
$$

By Biggins [4] it is known that $L_{k} / k \rightarrow \log \rho_{1}$ and $R_{k} / k \rightarrow \log \rho_{2}$ (almost surely). Hence, Theorem 2.2 is in some sense a refined version of Biggins' result. It also makes precise the ideas evocated in Kyprianou [15], section 2. In this context, the functions $w_{1}$ and $w_{2}$ are also called travelling waves. It is assumed that convergence to travelling waves is a much more general phenomenon, but there are only few examples where it is known (see Bachmann [2]). In section 5 we will show that a so-called intersection property will prove this almost automatically. Unfortunately it seems to be a non-trivial problem to verify this intersection property in a general setting. In Bachmann [2] this property was implicitly proved for iid $X_{j}$ with log-concave density. In the context of Theorem 2.2 (and also of Theorem 2.1) we can prove an intersection property on the level of Laplace transforms. We remark that the results in Bramson [8] on Brownian branching random walks rely on an intersection property, too. 
Binary (and more generally $m$-ary) search trees and their variants are one of the most popular data structures (see [17]). They also appear implicitly in the analysis of Quicksort. They become random trees by assuming proper random models on the input data. The most common model is the random permutation model, that is, one assumes that every permutation of $n$ input elements (the data) is equally likely.

In order to speed up Quicksort one also uses the median of $(2 t+1)$-variant. In every step one uses the median of $2 t+1$ random elements as the pivot. Of course, this gives rise to a more balanced binary search tree, the fringe balanced binary search tree. (The $m$-ary version is the so called fringe balanced $m$-ary search tree, where one has to build up $m-1$ pivots from $m(t+1)-1$ random elements.) Equivalently one can build up split trees (see [12]) with splitting probabilities for a tree storing $n$ items with $m$ subtrees with $n_{1}, n_{2}, \ldots, n_{m}$ items $\left(n_{1}+n_{2}+\cdots+n_{m}=n-m+1\right.$; the root stores $m-1$ items) of the form

$$
\frac{\left(\begin{array}{c}
n_{1} \\
t
\end{array}\right)\left(\begin{array}{c}
n_{2} \\
t
\end{array}\right) \cdots\left(\begin{array}{c}
n_{m} \\
t
\end{array}\right)}{\left(\begin{array}{c}
n \\
m(t+1)-1
\end{array}\right)} .
$$

For this random model of trees let $H_{n}^{(m, t)}$ denote the height and $\bar{H}_{n}^{(m, t)}$ the saturation level, that is the maximal level up to which the tree is a complete $m$-ary tree. For example, the splitting probabilities can be used to get an explicit recurrence of the form

$$
\begin{aligned}
& \operatorname{Pr}\left\{H_{n}^{(m, t)} \leq k+1\right\} \\
& =\sum_{n_{1}+n_{2}+\cdots+n_{m}=n-m+1} \frac{\left(\begin{array}{c}
n_{1} \\
t
\end{array}\right)\left(\begin{array}{c}
n_{2} \\
t
\end{array}\right) \cdots\left(\begin{array}{c}
n_{m} \\
t
\end{array}\right)}{\left(\begin{array}{c}
n \\
m(t+1)-1
\end{array}\right)} \operatorname{Pr}\left\{H_{n_{1}}^{(m, t)} \leq k\right\} \cdots \operatorname{Pr}\left\{H_{n_{m}}^{(m, t)} \leq k\right\} .
\end{aligned}
$$

A tree of size $n$ has height $\leq k+1$ if and only if all its subtrees (of sizes $n_{1}, \ldots, n_{m}$ ) have heights $\leq k$. Hence, the problem is to solve this recurrence in some sense.

The height $H_{n}=H_{n}^{(2,0)}$ of binary search trees (and its variants) has a long history (compare with [13]). In 1986 Devroye [10] proved that the expected value $\mathbf{E} H_{n}$ satisfies the asymptotic relation $\mathbf{E} H_{n} \sim c \log n$ (as $\left.n \rightarrow \infty\right)$, where $c=4.31107 \ldots$ is the largest real solution of the equation $\left(\frac{2 e}{c}\right)^{c}=e$. (Earlier Pittel [21] had shown that $H_{n} / \log n \rightarrow \gamma$ almost surely as $n \rightarrow \infty$, where $\gamma \leq c$, compare also with Robson [23].) Based on numerical data Robson conjectured that the variance Var $H_{n}$ is bounded. Eventually, Reed [22] and independently Drmota [13] settled Robson's conjecture and proved that

$$
\operatorname{Var} H_{n}=\mathcal{O}(1)
$$

In [13] the distribution of $H_{n}$ was also asymptotically determined.

The following theorem generalizes this result to the height (and saturation level) of fringe balanced $m$-ary search trees. 
Theorem 2.3 Let $m \geq 2$ and $t \geq 0$ be integers. There exist sequences $c_{k}, d_{k}$ with

$$
\lim _{k \rightarrow \infty} \frac{c_{k+1}}{c_{k}}=\rho_{1} \quad \text { and } \quad \lim _{k \rightarrow \infty} \frac{d_{k+1}}{d_{k}}=\rho_{2}
$$

such that

$$
\operatorname{Pr}\left\{H_{n}^{(m, t)} \leq k\right\}=F\left(n / c_{k}\right)+o(1) \quad \text { and } \quad \operatorname{Pr}\left\{\bar{H}_{n}^{(m, t)}>k\right\}=G\left(n / d_{k}\right)+o(1),
$$

where $F(x)$ and $G(x)$ are as in Theorem 2.1.

Furthermore, set $k_{1}(n)=\max \left\{k \geq 0: c_{k} \leq n\right\} \sim \log n / \log \rho_{1}$ and $k_{2}(n)=\max \{k \geq 0:$ $\left.d_{k} \leq n\right\} \sim \log n / \log \rho_{2}$. Then

$$
\mathbf{E} H_{n}^{(m, t)}=k_{1}(n)+O(1) \quad \text { and } \quad \mathbf{E} \bar{H}_{n}^{(m, t)}=k_{2}(n)+O(1)
$$

and there exists $\eta>0$ with

$$
\operatorname{Pr}\left\{\left|H_{n}^{(m, t)}-k_{1}(n)\right|>y\right\}=O\left(e^{-\eta y}\right) \quad \text { and } \quad \operatorname{Pr}\left\{\left|\bar{H}_{n}^{(m, t)}-k_{2}(n)\right|>y\right\}=O\left(e^{-\eta y}\right) .
$$

In particular we have, as $n \rightarrow \infty$,

$$
\operatorname{Var} H_{n}^{(m, t)}=O(1) \quad \text { and } \quad \operatorname{Var} \bar{H}_{n}^{(m, t)}=O(1) .
$$

These theorems show that the distribution of the longest part in the random multisection problem and the height of $m$-ary search trees (and some extensions) are closely related. This similarity has been already observed and used by Devroye $[10,11]$ to prove that $H_{n}^{(m, 0)} / \log n \rightarrow$ $1 / \log \rho_{1}$ a.s. He uses an infinite $m$-ary trees where the $m$ edges of each node are labeled by independent copies of $V_{1}, \ldots, V_{m}$ and the nodes $v$ by the product $V_{j_{1}} V_{j_{2}} \cdots V_{j_{h}}$ corresponding to the path joining the root and $v$. The height $\tilde{H}_{n}$ of the random subtree consisting of the nodes with $V_{j_{1}} V_{j_{2}} \cdots V_{j_{h}} \leq 1 / n$ corresponds to the leftmost particle in the branching random walk determined by the point process $Z=\delta_{-\log V_{1}}+\cdots+\delta_{-\log V_{m}}$ or to the largest fragment in the corresponding random multisection problem, more precisely

$$
\operatorname{Pr}\left\{\tilde{H}_{n} \leq k\right\}=\operatorname{Pr}\left\{L_{k}>\log n\right\}=\bar{P}_{k}(n) .
$$

Hence by restating Biggin's result [4] properly it follows that $\tilde{H}_{n} / \log n \rightarrow 1 / \log \rho_{1}$ a.s. Finally, the distribution of $H_{n}^{(m, 0)}$ can be compared to that of $\tilde{H}_{n}$, for example one has $\operatorname{Pr}\left\{H_{n}^{(m, 0)} \geq\right.$ $k\} \geq \operatorname{Pr}\left\{\tilde{H}_{n} \geq k\right\}$. Thus one gets $H_{n}^{(m, 0)} / \log n \rightarrow 1 / \log \rho_{1}$, too. By the way, the saturation level corresponds to the rightmost particle or to the shortest fragment. Hence, by following exactly Devroye's lines we immediately get

$$
\frac{H_{n}^{(m, t)}}{\log n} \rightarrow \frac{1}{\log \rho_{1}} \text { a.s. and } \quad \frac{\bar{H}_{n}^{(m, t)}}{\log n} \rightarrow \frac{1}{\log \rho_{2}} \text { a.s. }
$$


In view of this discussion, Theorem 2.3 is a refinement of this first approximation.

The philosophy of Devroye relies on a coupling method between $\tilde{H}_{n}$ and $H_{n}^{(m, 0)}$. Fundamentally, it works because the two models can be considered on the same probability space: by embedding the search tree process in continuous time, (a) the $m$-ary search tree is the continuous tree process observed at discrete random stopping times; (b) the multisection branching random walk (or the fragmentation process) is constructed from the continuous time process, as being the asymptotic proportions in the subtrees. For details, see $[9,1]$. Consequently, formal similarities appear on the Laplace transforms. In particular, the Laplace transform of $\bar{P}_{k}$ satisfies the same (recursive) differential equations as the generating functions of the probabilities $\operatorname{Pr}\left\{H_{n}^{(m, t)} \leq k\right\}$, compare with (16) and (42). Luckily this formal similarity also leads to a similar way for the asymptotic analysis. Both problems can be described with the help of a common travelling wave $F(x)$. The crucial property for the proof is again an intersection property, see Lemma 4.12.

Although there is a strong formal similarity, we decided to treat both problems separately. First, the random multisection problem is easier since it is only discrete in the number of steps $k$ whereas the height problem is discrete in the number of nodes $n$ and the level $k$. This causes additional monotonicity considerations and also additional approximation steps. Further, in the random multisection problem one is mainly interested in the asymptotics $k \rightarrow \infty$ whereas in the tree problem one looks as $n \rightarrow \infty$.

The structure of the paper is the following one. In Section 3 we prove the existence of a travelling wave with the help of a stochastic fixed point equation. The main part is Section 4, where we prove convergence to a travelling wave (Theorem 2.1 and 2.2) and the analogue for $m$-ary search trees (Theorem 2.3). Finally, in Section 5 we discuss the implications of the intersection property in the context of branching random walks.

\section{Existence of a Travelling Wave}

In this section we consider the functional equation

$$
F(x / \rho)=\mathbf{E}\left(F\left(x V_{1}\right) \cdots F\left(x V_{m}\right)\right)
$$

for critical values $\rho>1$ and show that there is (up to scaling) a unique solution that can be interpreted as a travelling wave solution of a certain branching random walk. In fact, we will present two proofs. The first one is based on known results on the fixed point solution of the equation

$$
Y=\sum_{i \geq 1} A_{i} Y_{i}
$$

where $\left(A_{1}, A_{2}, \ldots\right)$ is a random decreasing sequence of non-negative numbers that ultimatively vanish and $Y_{i}$ are copies of a random variable $Y$ that are independent of each other and $\left(A_{1}, A_{2}, \ldots\right)$. 
The second proof will be presented in the next section and follows from an analysis based on the Laplace transform. We remark that the first approach is much more general than the second one but the second approach provides tail estimates for the solution of (6) that are not granted by the first method.

Alternatively to (7) one usually considers the Laplace transform $\Phi(x)=\mathbf{E} e^{-Y x}$ and the corresponding equation

$$
\Phi(x)=\mathbf{E}\left(\prod_{i \geq 1} \Phi\left(x A_{i}\right)\right) \quad(x \geq 0)
$$

and searches for solutions $\Phi$ of (8), in the set of Laplace transforms of finite non-negative random variables. Note that $\Phi(a x)$ is also a solution of (8) for every $a>0$ if $\Phi(x)$ satisfies (8). There is an extensive literature that deals with the problem of establishing the uniqueness of solutions for equations like (8). For instance the work by Biggins and Kyprianou [7], Liu [16] and already of Durrett and Liggett [14] for the nonrandom case where $N \equiv m$ show that, under suitable conditions, (8) has (up to this scaling) a unique solution:

Proposition 3.1 Set $v(\alpha)=\log \left(\mathbf{E}\left(\sum_{i \geq 1} A_{i}^{\alpha}\right)\right)$ and suppose that $v(0)>0$, that $\alpha=1$ is contained in the interior of $\{\alpha: v(\alpha)<\infty\}$, and that $v(1)=v^{\prime}(1)=0$ (critical case). Then the equation (8) has (up to scaling) a unique solution $\Phi(x)$ in the set of Laplace transform of non-negative random variables. This solution is such that

$$
\lim _{x \rightarrow 0+} \frac{1-\Phi(x)}{-x \log x}=c_{1}
$$

exists (with a positive constant $c_{1}$ ).

It should be noted that the non critical case $v(1)=0, v^{\prime}(1)<0$ is much easier to handle. In Biggins and Kyprianou [5] it is shown that there is also a unique solution.

Before stating our result we need the following property.

Lemma 3.2 Let $m \geq 2$ and $t \geq 0$ be integers. Then there exist exactly two solutions $\beta_{1}>0$ and $\beta_{2}<0$ of the equation

$$
\sum_{j=0}^{(m-1)(t+1)-1} \log (\beta+t+1+j)-\log \left(\frac{(m(t+1)) !}{(t+1) !}\right)=\sum_{j=0}^{(m-1)(t+1)-1} \frac{\beta}{\beta+t+1+j} .
$$

Set

$$
\rho_{1}=\exp \left(\sum_{j=0}^{(m-1)(t+1)-1} \frac{1}{\beta_{1}+t+1+j}\right) \quad \text { and } \quad \rho_{2}=\exp \left(\sum_{j=0}^{(m-1)(t+1)-1} \frac{1}{\beta_{2}+t+1+j}\right) .
$$

Then $1<\rho_{1}<\rho_{2}$.

Furthermore, 
1. if $1<\rho<\rho_{1}$ then the equation

$$
\frac{(m(t+1)) !}{(t+1) !} \rho^{\beta}=(\beta+t+1)(\beta+t+2) \cdots(\beta+m(t+1)-1)
$$

has exactly two solutions $\beta^{\prime}, \beta^{\prime \prime}$ with $0<\beta^{\prime}<\beta_{1}<\beta^{\prime \prime}$,

2. if $\rho=\rho_{1}$, then (11) has exactly one solution $\beta=\beta_{1}$,

3. if $\rho_{1}<\rho<\rho_{2}$ then (11) has no solutions,

4. if $\rho=\rho_{2}$, then (11) has exactly one solution $\beta=\beta_{1}$, and

5. if $\rho>\rho_{2}$ then (11) has exactly two solutions $\beta^{\prime}, \beta^{\prime \prime}$ with $-t-1<\beta^{\prime}<\beta_{2}<\beta^{\prime \prime}<0$.

For the reader's convenience we provide a short proof.

Proof. For $\beta>-t-1$ set

$$
\bar{v}(\beta)=\log \left(\frac{(m(t+1)) !}{(t+1) !}\right)-\sum_{j=0}^{(m-1)(t+1)-1} \log (\beta+t+1+j) .
$$

Then $\bar{v}(\beta)$ is strictly convex and we have $\bar{v}(0)=\log m>0$ and $\bar{v}(1)=0$. Thus, the graph of $\bar{v}(\beta)$ is the boundary $\partial C=\{(\beta, \bar{v}(\beta)): \beta>-t-1\}$ of a strictly convex region $C=\{(\beta, \gamma)$ : $\beta>-t-1, \gamma \geq \bar{v}(\beta)\}$ that does not contain the origin $(0,0)$ but we have $(0, \log m) \in \partial C$ and $(1,0) \in \partial C$.

Now note that (10) is equivalent to $\bar{v}(\beta) / \beta=\bar{v}^{\prime}(\beta)$. In geometric terms this means that one is looking at tangents to the convex region $C$ that contain the origin $(0,0)$. Since $C$ is strictly convex there are exactly two tangents $\gamma=\beta \log \frac{1}{\rho_{1}}$ and $\gamma=\beta \log \frac{1}{\rho_{2}}$ with $1<\rho_{1}<\rho_{2}$ that correspond to the tangent points $\left(\beta_{1}, \bar{v}\left(\beta_{1}\right)\right)$ and $\left(\beta_{2}, \bar{v}\left(\beta_{2}\right)\right)$ with $\beta_{1}>0$ and $-t-1<\beta_{2}<0$.

Finally, (11) is equivalent to $\bar{v}(\beta)=\beta \log \frac{1}{\rho}$ or to the intersection points of the boundary of $C$ and the line $\gamma=\beta \log \frac{1}{\rho}$. By obvious convexity arguments this completes the proof of the lemma.

With the help of Proposition 3.1 we can easily prove the following property.

Theorem 3.3 Let $m \geq 2$ and $t \geq 0$ be integers and $\left(V_{1}, \ldots, V_{m}\right)$ be $t$-beta-distributed. Then for $\rho=\rho_{1}$ and $\rho=\rho_{2}$ as defined in Lemma 3.2 the functional equation (6) has (up to scaling) a unique solution $F_{1}(x)$ resp. $F_{2}(x)$. This solution is such that

$$
1-F_{1}(x) \sim d_{1} x^{\beta_{1}} \log x \quad(x \rightarrow 0+)
$$

and

$$
1-F_{2}(x) \sim d_{2} x^{\beta_{2}} \log x \quad(x \rightarrow \infty)
$$


for non-zero real constants $d_{1}, d_{2}$. Furthermore, $F_{1}(x)$ and $F_{2}(x)$ are strictly decreasing resp. increasing, continuous, and satisfy

$$
\lim _{x \rightarrow \infty} F_{1}(x)=\lim _{x \rightarrow 0+} F_{2}(x)=0 .
$$

Proof. For $1 \leq i \leq m$ set $A_{i}=\left(\rho_{1} V_{i}\right)^{\beta_{1}}$. Then

$$
v(\alpha)=\alpha \beta_{1} \log \rho_{1}+\log (m t !)-\sum_{j=0}^{t} \log \left(\alpha \beta_{1}+t+j+1\right)
$$

and by (10) and (11) we obtain directly that $v(1)=v^{\prime}(1)=0$. Using Proposition 3.1 it follows that there exists a function $\Phi_{1}(x)$ that is unique up to scaling and satisfies

$$
\Phi_{1}(x)=\mathbf{E}\left(\prod_{i=1}^{m} \Phi_{1}\left(x\left(\rho_{1} V_{1}\right)^{\beta_{1}}\right)\right)
$$

and $1-\Phi_{1}(x) \sim-d_{1} x \log x$ as $x \rightarrow 0+$, for an appropriate constant $d_{1}>0$. Hence, $F_{1}(x)=$ $\Phi_{1}\left(x^{\beta_{1}}\right)$ satisfies the functional equation (6) and (12). Since $\Phi_{1}(x)=\mathbf{E}\left(e^{-Y x}\right)$ is the Laplace transform of a non-negative random variable $Y$, it is decreasing. Furthermore, $\Phi_{1}^{\prime}(x)=-\mathbf{E}\left(Y e^{-Y x}\right)$ $<0$. Thus $\Phi_{1}(x)$ and $F_{1}(x)$ are strictly decreasing (and continuous). It also follows that the limit $D=\lim _{x \rightarrow \infty} F_{1}(x) \geq 0$ exists. By (12) we know that $D<1$ and from (6) it follows that $D=D^{m}$. Consequently $D=0$.

In completely the same way we can proceed with $\rho_{2}$ and $\beta_{2}<0$ and obtain corresponding properties for $F_{2}(x)$.

Remark 3.4 As already mentioned above, using Proposition 3.1, we can only get one-sided tail estimates for $F_{1}(x)$ and $F_{2}(x)$.

Remark 3.5 The functional equation (6) has also unique solutions for $1<\rho<\rho_{1}$ and for $\rho>\rho_{2}$. This case corresponds to $v(1)=0$ and $v^{\prime}(1)<0$.

Remark 3.6 The solution of the functional equation (8) is the Laplace transform of a random variable which has a meaning in the branching random walk framework (see [6, 7, 15]): let $Z=\delta_{X_{1}}+\cdots+\delta_{X_{N}}$ be the point process driving the branching random walk with $N \equiv m$ and for $j=1,2, \ldots, m, X_{j}=\log \left(1 / V_{j}\right)$. Call $X_{u}$ the position of a particle $u$ (the sum of i.i.d. displacements in the random walk on the branch leading to u). Let

$$
\bar{m}(\beta)=\mathbf{E}\left(\sum_{j=1}^{N} e^{-\beta X_{j}}\right)
$$


and

$$
W_{n}(\beta)=\sum_{|u|=n} \frac{e^{-\beta X_{u}}}{\bar{m}(\beta)^{n}}=\sum_{|u|=n} e^{-\beta\left(X_{u}+n \frac{\log \bar{m}(\beta)}{\beta}\right)}
$$

be the additive martingale associated to the branching random walk. The critical case corresponds to the case where $\beta$ is a solution of the equation

$$
\frac{\log \bar{m}(\beta)}{\beta}=\frac{\bar{m}^{\prime}(\beta)}{\bar{m}(\beta)},
$$

and there are two solutions $\beta_{1}$ and $\beta_{2}$ of this equation. In our fragmentation case, it appears that it is the same equation as equation (10) in lemma 3.2. ${ }^{3}$ So call them $\beta_{1}>0$ and $\beta_{2}<0$. For $\beta_{1}$ for instance (it is the same with $\left.\beta_{2}\right), W_{n}\left(\beta_{1}\right)$ tends to 0 almost surely when $n$ goes to infinity, giving a trivial solution to the fixed point equation. Besides the derivative martingale (so called because it can be derived by differentiating the additive martingale with respect to $\beta$ ) is defined by

$$
W_{n}^{\prime}(\beta)=-\sum_{|u|=n}\left(X_{u}+n \frac{\bar{m}^{\prime}(\beta)}{\bar{m}(\beta)}\right) \frac{e^{-\beta X_{u}}}{\bar{m}(\beta)^{n}} .
$$

In the critical case, it has almost surely a finite limit $W^{\prime}\left(\beta_{1}\right)$ but with an infinite expectation. It is a solution of the same fixed point equation and its Laplace transform is a solution of (8), so that the wave can be represented as

$$
F_{1}(x)=\Phi_{1}\left(x^{\beta_{1}}\right)=\mathbf{E}\left(e^{-x^{\beta_{1}} W^{\prime}\left(\beta_{1}\right)}\right)
$$

Of course, $W^{\prime}\left(\beta_{1}\right)$ coincides with $Y$ in the proof of Theorem 3.3.

\section{Convergence to Travelling Wave}

In this section $\left(V_{1}, \ldots, V_{m}\right)$ will always denote a $t$-beta distributed random vector with a nonnegative integer parameter $t$.

\subsection{Random Fragmentation Problem}

In what follows we will prove Theorems 2.1 and 2.2. One major tool is the property of Lemma 4.2 that we call intersection property. It transfers inequalities from one step to the next. Convergence to a travelling wave (resp. to its Laplace transform) is then almost automatic, see Lemmas 4.3 and 4.4 .

\footnotetext{
${ }^{3}$ The function $\bar{v}(\beta)$ in the proof of Lemma 3.2 equals $\log \bar{m}(\beta)$ for a $t$-beta distributed random vector $\left(V_{1}, \ldots, V_{m}\right)$.
} 
Lemma 4.1 Suppose that $F(x), x \geq 0$, is a non-negative bounded function and let

$$
L_{F}(u)=\int_{0}^{\infty} e^{-x u} F(x) d x \quad(u>0)
$$

be the Laplace transform of $F(x)$. Furthermore, let $T F$ be given by

$$
(T F)(x)=\mathbf{E}\left(F\left(x V_{1}\right) \cdots F\left(x V_{m}\right)\right) .
$$

Then $^{4}$

$$
L_{T F}^{(m(t+1)-1)}(u)=(-1)^{m-1} \frac{(m(t+1)-1) !}{(t !)^{m}}\left(L_{F}^{(t)}(u)\right)^{m} .
$$

Proof. The proof is easy and is left to the reader.

Lemma 4.2 Let $m \geq 2$ and $t \geq 0$ be given integers and suppose that $F(x)$ and $G(x)$ are non-negative functions, that are defined for $x \geq 0$, have the following properties:

1. The Laplace transforms $L_{F}(u)$ and $L_{G}(u)$ exist for $u>u_{c}$ (for some $u_{c} \geq-\infty$ ).

2. The $t$-th derivative $\hat{\Delta}^{(t)}(u)$ of the difference $\hat{\Delta}(u)=L_{F}(u)-L_{G}(u)$ exactly one zero $u_{0}>u_{c}$ such that $(-1)^{t} \hat{\Delta}^{(t)}(u)>0$ for $u>u_{0}$.

3. There exists $u_{1}$ such that $(-1)^{j} \hat{\Delta}_{1}^{(j)}(u)>0$ for $u>u_{1}$ and $j=0,1, \ldots, m(t+1)-1$, where $\hat{\Delta}_{1}(u)=L_{T F}(u)-L_{T G}(u)$.

Then the differences

$$
\hat{\Delta}_{1}^{(j)}(u)=L_{T F}^{(j)}(u)-L_{T G}^{(j)}(u)
$$

have at most one zero $>u_{c}$ for $j=0,1, \ldots, m(t+1)-1$.

Proof. By (16) we have

$$
\hat{\Delta}_{1}^{(m(t+1)-1)}(u)=(-1)^{m-1} \frac{(m(t+1)-1) !}{(t !)^{m}} \hat{\Delta}_{1}^{(t)}(u) \sum_{\ell=0}^{m-1}\left(L_{F}^{(t)}(u)\right)^{\ell}\left(L_{G}^{(t)}(u)\right)^{m-1-\ell} .
$$

Since $(-1)^{\ell} L_{F}^{(\ell)}(u)>0$ and (similarly for $\left.L_{G}(u)\right)$ it follows that $\hat{\Delta}_{1}^{(m(t+1)-1)}(u)$ has exactly one zero $u_{0}>u_{c}$ and that

$$
(-1)^{m(t+1)-1} \hat{\Delta}_{1}^{(m(t+1)-1)}(u)>0
$$

for $u>u_{0}$.

Now, observe that by assumption $(-1)^{m(t+1)-2} \hat{\Delta}_{1}^{(m(t+1)-2)}(u)>0$ for $u \geq u_{1}$. Hence, if we consider the mapping

$$
v \mapsto f(v)=(-1)^{m(t+1)-2} \hat{\Delta}_{1}^{(m(t+1)-2)}\left(u_{1}-v\right) \quad\left(0 \leq v<u_{1}-u_{c}\right)
$$

\footnotetext{
${ }^{4}$ We use the notation $f^{(t)}(x)$ for the $t$-th derivative of $f(x)$.
} 
then it follows that $f(0)>0$, that $f(v)$ is increasing for $0 \leq v \leq u_{1}-u_{0}$ and decreasing for $u_{1}-u_{0} \leq v<u_{1}-u_{c}$. Thus, $f(v)$ has at most one zero for $0 \leq v \leq u_{1}-u_{c}$. Consequently, $\hat{\Delta}_{1}^{(m(t+1)-2)}(u)$ has at most one zero $>u_{c}$.

In the same way we can proceed further. If $(-1)^{m(t+1)-2} \hat{\Delta}_{1}^{(m(t+1)-2)}(u)$ has no zero $>u_{c}$ then it follows that $(-1)^{m(t+1)-3} \hat{\Delta}_{1}^{(m(t+1)-3)}(u)>0$ for all $u>u_{c}$ (and so on). If $(-1)^{m(t+1)-2} \hat{\Delta}_{1}^{(m(t+1)-2)}(u)$ has a zero $>u_{c}$ then we obtain that $\hat{\Delta}_{1}^{(m(t+1)-3)}(u)$ has at most one zero $>u_{c}$. The lemma follows now by induction.

Lemma 4.3 Let $F_{0}(x)$ be defined by

$$
F_{0}(x)= \begin{cases}1 & \text { for } 0 \leq x<1 \\ 0 & \text { for } x \geq 1\end{cases}
$$

and recursively $F_{k+1}=T F_{k}$ for $k \geq 0$. Then there exist $u_{k}>0$ satisfying

$$
\frac{1}{u_{k}} L_{F_{k}}\left(\frac{1}{u_{k}}\right)=\frac{1}{2}
$$

with

$$
\begin{aligned}
& \frac{1}{u_{k+1}} L_{F_{k+1}}\left(\frac{u}{u_{k+1}}\right) \geq \frac{1}{u_{k}} L_{F_{k}}\left(\frac{u}{u_{k}}\right) \quad(\text { for } u<1), \\
& \frac{1}{u_{k+1}} L_{F_{k+1}}\left(\frac{u}{u_{k+1}}\right) \leq \frac{1}{u_{k}} L_{F_{k}}\left(\frac{u}{u_{k}}\right) \quad(\text { for } u>1) .
\end{aligned}
$$

Note that $F_{k}(x)$ equals $\bar{P}_{k}(x)$ from Theorem 2.1 .

Proof. First of all we note that the Laplace transform $L_{F_{k}}(u)$ is defined for all (real and complex) $u$. For example, $L_{F_{0}}(u)=\left(1-e^{-u}\right) / u$. The functions $L_{F_{k}}(u)$ can be recursively calculated with the help of (16).

Since $F_{1}(x)=1$ for $0 \leq x \leq 1$ and $F_{1}(x)>0$ for $x>1$ we surely have $F_{1}(x) \geq F_{0}(x)$ for $x \geq 0$ (and $F_{1}(x)>F_{0}(x)$ for $x>1$ ). Consequently we obtain by induction $F_{k+1}(x) \geq F_{k}(x)$ (and $F_{k+1}(x)>F_{k}(x)$ for $x>1$ ). This implies that $L_{F_{k+1}}(u)>L_{F_{k}}(u)$ for $u>0$. Since $L_{-F_{k}^{\prime}}(u)=1-u L_{F_{k}}(u)$ we also have $L_{-F_{k+1}^{\prime}}(u)<L_{-F_{k}^{\prime}}(u)$.

Note that $L_{-F_{k}^{\prime}}(u) \sim 1$ as $u \rightarrow 0+$, that $L_{-F_{k}^{\prime}}(u) \rightarrow 0$ as $u \rightarrow \infty$, and that $L_{-F_{k}^{\prime}}(u)$ is strictly decreasing. Hence, there uniquely exists $u_{k}>0$ with $L_{-F_{k}^{\prime}}\left(1 / u_{k}\right)=\frac{1}{2}$ and consequently

$$
\frac{1}{u_{k}} L_{F_{k}}\left(\frac{1}{u_{k}}\right)=\frac{1}{2}
$$

We also have $u_{k+1}>u_{k}$ since $L_{-F_{k+1}^{\prime}}(u)<L_{-F_{k}^{\prime}}(u)$ and $L_{-F_{k}^{\prime}}(u)$ is strictly decreasing.

Now fix some $k \geq 0$ and set (for $j \geq 0$ )

$$
\tilde{F}_{j}(x)=F_{j}\left(x u_{k}\right) \text { and } \tilde{G}_{j}(x)=F_{j+1}\left(x u_{k+1}\right) .
$$


Then $T \tilde{F}_{j}=\tilde{F}_{j+1}, T \tilde{G}_{j}=\tilde{G}_{j+1}$, and

$$
L_{\tilde{F}_{k}}(1)=L_{\tilde{G}_{k}}(1)=\frac{1}{2} .
$$

For convenience, set $\Delta_{j}(x)=\tilde{F}_{j}(x)-\tilde{G}_{j}(x)$ and $\hat{\Delta}_{j}(u)=L_{\Delta_{j}}(u)$. By construction we have $\Delta_{j}(x)=0$ for $0 \leq x \leq 1 / u_{k+1}$ and $\Delta_{j}(x)>0$ for $1 / u_{k+1}<x \leq 1 / u_{k}$. Thus, there exists $u_{j}^{\prime}>0$ such that

$$
(-1)^{\ell} \hat{\Delta}^{(\ell)}(u)=\int_{0}^{\infty} \Delta_{j}(x) x^{\ell} e^{-x u} d x=(-1)^{\ell}\left(L_{\tilde{F}_{j}}^{(\ell)}(u)-L_{\tilde{G}_{j}}^{(\ell)}(u)\right)>0
$$

for $u \geq u_{j}^{\prime}$ and $\ell=0,1, \ldots, m(t+1)-1$. We now show inductively that the differences

$$
\hat{\Delta}_{j}^{(\ell)}(u)=L_{\tilde{F}_{j}}^{(\ell)}(u)-L_{\tilde{G}_{j}}^{(\ell)}(u)
$$

have exactly one (positive) zero for $j=0,1, \ldots, k$ and $\ell=0,1, \ldots, m(t+1)-1$. This is obviously true for $j=0$ since $\Delta_{0}(x)$ has exactly one "zero". (Note that $u_{k+1}>u_{k}$ !) Now, assume that the functions $\hat{\Delta}_{j}^{(\ell)}(u)(\ell=0,1, \ldots, k)$ have exactly one positive zero $u_{j, \ell}^{\prime \prime}$ (for some $j<k$ ). Since $(-1)^{\ell} \hat{\Delta}_{j}^{(\ell)}(u)$ is positive for $u \geq u_{j}^{\prime}$ we know that $(-1)^{\ell} \hat{\Delta}_{j}^{(\ell)}(u)<0$ for $0<u<u_{j, \ell}^{\prime \prime}$ and $(-1)^{\ell} \hat{\Delta}_{j}^{(\ell)}(u)>0$ for $u>u_{j, \ell}^{\prime \prime}$. From $(20)$ we also know that $(-1)^{\ell} \hat{\Delta}_{j+1}^{(\ell)}(u)>0$ for $u>u_{j+1}^{\prime}$ and $\ell=0,1, \ldots, m(t+1)-1$. Thus, we can apply Lemma 4.2 and obtain that $\hat{\Delta}_{j+1}^{(\ell)}(u)(\ell=$ $0,1, \ldots, m(t+1)-1)$ have at most one positive zero. Hence, it remains to show that there is some zero. If $\hat{\Delta}_{j+1}^{\left(\ell_{0}\right)}(u)$ has no zero (for some $\ell_{0}$ ) then it follows that $\hat{\Delta}_{j+1}^{(\ell)}(u)$ have no zero for $\ell=0,1, \ldots, \ell_{0}$. In particular it follows then by induction (with the help of the methods of Lemma 4.1$)$ that $\hat{\Delta}_{j+1}(u)>0, \hat{\Delta}_{j+2}(u)>0, \ldots, \hat{\Delta}_{k}(u)>0$ for all $u>0$. This contradicts $\hat{\Delta}_{k}(1)=0$ and consequently $\hat{\Delta}_{j+1}^{(\ell)}(u)$ has exactly one positive zero for all $\ell=0,1, \ldots, m(t+1)-1$.

We also know now that $u=1$ is the only zero of $\hat{\Delta}_{k}(1)=0$. By combining this with $(20)$ we have $\hat{\Delta}_{k}(u)<0$ for $u<1$ and $\hat{\Delta}_{k}(u)>0$ for $u>1$. Of course, this is just a translation of (17) and (18) since $L_{\tilde{F}_{k}}(u)=\frac{1}{u_{k}} L_{F_{k}}\left(\frac{u}{u_{k}}\right)$ and $L_{\tilde{G}_{k}}(u)=\frac{1}{u_{k+1}} L_{F_{k+1}}\left(\frac{u}{u_{k+1}}\right)$

Lemma 4.4 Let $F_{k}(x)$ and $u_{k}$ be defined as in Lemma 4.3. Then the limit

$$
L_{1}(u)=\lim _{k \rightarrow \infty} \frac{1}{u_{k}} L_{F_{k}}\left(\frac{u}{u_{k}}\right)
$$

exists for each real $u$ and $L_{1}(u)$ satisfies the differential equation

$$
L_{1}^{(m(t+1)-1)}(u)=(-1)^{m-1} \frac{(m(t+1)-1) !}{(t !)^{m}}\left(\frac{1}{\rho_{1}^{t+1}} L_{1}^{(t)}\left(u / \rho_{1}\right)\right)^{m},
$$


where $\rho_{1}$ is as defined in Lemma 3.2. We also have

$$
\rho_{1}=\lim _{k \rightarrow \infty} \frac{u_{k+1}}{u_{k}}
$$

and

$$
\log u_{k}=k \log \rho_{1}+\Theta(\log k) \quad(k \rightarrow \infty) .
$$

Furthermore, $L_{1}(u)$ is the Laplace transform of a distribution function $F(x)$ that satisfies the fixed point equation

$$
F\left(x / \rho_{1}\right)=\mathbf{E}\left(F\left(x V_{1}\right) \cdots F\left(x V_{m}\right)\right),
$$

that is, $F(x)$ equals (up to scaling) $F_{1}(x)$ from Lemma 3.2, and we have

$$
F_{k}(x)=F\left(x / u_{k}\right)+o(1)
$$

uniformly for $x \geq 0$, as $k \rightarrow \infty$.

Proof. From (17) and (18) it directly follows that the limit $L_{1}(u)$ in (21) exists. Furthermore, the function $1-u L_{1}(u)$ is the limit of the moment generating function of a certain sequence of continuous random variables $Y_{k}$ with distribution function $1-F_{k}\left(x u_{k}\right)$. Consequently, $1-u L_{1}(u)$ equals the moment generating function of a random variable $Y$ that is the weak limit of the $Y_{k}$ 's. Of course, the distribution function $1-F(x)$ of $Y$ satisfies $F(x)=F_{k}\left(x u_{k}\right)+o(1)$ as $k \rightarrow \infty$ for all $x$ that are continuity points of $F$.

Since $L_{1}(u)$ is a continuous function and the convergence to $L_{1}(u)$ is monotone, it follows that the convergence $\left(1 / u_{k}\right) L_{F_{k}}\left(u / u_{k}\right) \rightarrow L_{1}(u)$ is uniform in $u$ over intervals of the form $\left[u^{\prime}, u^{\prime \prime}\right]$ with $0<u^{\prime}<u^{\prime \prime}<\infty$. Hence, by elementary means it follows that all derivatives $\left(1 / u_{k}^{t+1}\right) L_{F_{k}}^{(t)}\left(u / u_{k}\right)$ converge uniformly to $L_{1}^{(t)}(u)$ for $u \in\left[u^{\prime}, u^{\prime \prime}\right]$, too.

Now we rewrite (16) to

$$
\frac{1}{u_{k+1}^{m(t+1)}} L_{F_{k+1}}^{(m(t+1)-1)}\left(\frac{u}{u_{k+1}}\right)=(-1)^{m-1} \frac{(m(t+1)-1) !}{(t !)^{m}}\left(\frac{1}{\kappa_{k}^{t+1}} \frac{1}{u_{k}^{t+1}} L_{F_{k}}^{(t)}\left(\frac{1}{\kappa_{k}} \frac{u}{u_{k}}\right)\right)^{m},
$$

where $\kappa_{k}=u_{k+1} / u_{k} \geq 1$. We can take the limit as $k \rightarrow \infty$ on both sides and observe (due to uniform convergence) that the limit

$$
\lim _{k \rightarrow \infty} \frac{1}{\kappa_{k}^{t+1}} L_{1}^{(t)}\left(\frac{u}{\kappa_{k}}\right)
$$

exists. It is now easy to conclude that $\kappa_{k}$ is a convergent sequence. Namely, if $\kappa_{k}$ had two different limit points $\tau_{1} \neq \tau_{2}$ then $L_{1}(u)$ would satisfy (22) with $\rho_{1}=\tau_{1}$ and $\rho_{1}=\tau_{2}$. This would imply $\tau_{1}^{m(t+1)} L_{1}^{(m(t+1)-1)}\left(\tau_{1} u\right)=\tau_{2}^{m(t+1)} L_{1}^{(m(t+1)-1)}\left(\tau_{2} u\right)$ or $F\left(x / \tau_{1}\right)=F\left(x / \tau_{2}\right)$ (for all continuity points of $F$ ) which is impossible since $F$ is decreasing and not constant. Thus, $\kappa_{k}=u_{k+1} / u_{k} \rightarrow \rho$ 
for some $\rho>1$. The case $\rho=1$ can be discharged because this case corresponds to $L_{1}(u)=1 / u$ or $F(x)=1$.

Next, we use the interpretation of Theorem 2.2 , that is, $1-F_{k}\left(e^{x}\right)$ can be interpreted as the distribution function of the leftmost particle $L_{k}$ of a corresponding discrete branching random walk that satisfies $L_{k} / k \rightarrow \log \rho_{1}$ almost surely (as $k \rightarrow \infty$ ). Thus, $\rho$ has to be equal to $\rho_{1}$, that is, (23). Further, (24) follows from [20].

Finally, it also follows that $F(x)$ solves (25). Thus, $F(x)$ equals (up to scaling) $F_{1}(x)$ from Theorem 3.3. Since $F_{1}(x)$ is continuous, (26) holds uniform for all $x \geq 0$.

In completely the same way we obtain the following properties.

Lemma 4.5 Let $G_{0}(x)$ be defined by

$$
G_{0}(x)= \begin{cases}0 & \text { for } 0 \leq x<1 \\ 1 & \text { for } x \geq 1\end{cases}
$$

and recursively $G_{k+1}=T G_{k}$ for $k \geq 0$. Furthermore, let $v_{k}>0$ be given by

$$
\frac{1}{v_{k}} L_{G_{k}}\left(\frac{1}{v_{k}}\right)=\frac{1}{2}
$$

Then we have

$$
\begin{array}{ll}
\frac{1}{v_{k+1}} L_{G_{k+1}}\left(\frac{u}{v_{k+1}}\right) \leq \frac{1}{v_{k}} L_{G_{k}}\left(\frac{u}{v_{k}}\right) & (\text { for } 0<u<1,) \\
\frac{1}{v_{k+1}} L_{G_{k+1}}\left(\frac{u}{v_{k+1}}\right) \geq \frac{1}{v_{k}} L_{G_{k}}\left(\frac{u}{v_{k}}\right) & (\text { for } u>1 .)
\end{array}
$$

We only observe that $G_{k}(x)=0$ for $0 \leq x \leq m^{k}$ and that $v_{k+1}>m v_{k}$. Observe also that $G_{k}(x)$ equals $\bar{Q}_{k}(x)$ from Theorem 2.1 and that $L_{G_{k}}(u)$ is only defined for (real) $u>0$.

Lemma 4.6 Let $G_{k}(x)$ and $v_{k}$ be defined as in Lemma 4.5. Then the limit

$$
L_{2}(u)=\lim _{k \rightarrow \infty} \frac{1}{v_{k}} L_{G_{k}}\left(\frac{u}{v_{k}}\right)
$$

exists for each $u>0$ and $L_{2}(u)$ satisfies the differential equation

$$
L_{2}^{(m(t+1)-1)}(u)=(-1)^{m-1} \frac{(m(t+1)-1) !}{(t !)^{m}}\left(\frac{1}{\rho_{2}^{t+1}} L_{2}^{(t)}\left(u / \rho_{2}\right)\right)^{m},
$$

where $\rho_{2}$ is as defined in Lemma 3.2. We also have

$$
\rho_{2}=\lim _{k \rightarrow \infty} \frac{v_{k+1}}{v_{k}}
$$


and

$$
\log v_{k}=k \log \rho_{2}+\Theta(\log k) \quad(k \rightarrow \infty) .
$$

Furthermore, $L_{2}(u)$ is the Laplace transform of a distribution function $G(x)$ that satisfies the fixed point equation

$$
G\left(x / \rho_{2}\right)=\mathbf{E}\left(G\left(x V_{1}\right) \cdots G\left(x V_{m}\right)\right),
$$

that is, $G(x)$ equals (up to scaling) $F_{2}(x)$ from Lemma 3.2, and we have

$$
G_{k}(x)=G\left(x / v_{k}\right)+o(1)
$$

uniformly for $x \geq 0$, as $k \rightarrow \infty$.

With the help of Lemma 4.4 and Lemma 4.6 we have proved almost entirely the first part of Theorems 2.1 and 2.2. In order to complete the proofs we have to show that $u_{k}$ and $x_{k}$ (resp. $v_{k}$ and $\left.y_{k}\right)$ are related in a proper way and to provide proper tail estimates.

Lemma 4.7 Let $x_{k}, y_{k}, u_{k}, v_{k}$ be as defined in Theorem 2.1 and Lemmas 4.3 and 4.5. Then there exist positive constants $C_{1}, C_{2}$ such that the following limiting relations hold:

$$
\lim _{k \rightarrow \infty} \frac{u_{k}}{x_{k}}=C_{1} \quad \text { and } \quad \lim _{k \rightarrow \infty} \frac{v_{k}}{y_{k}}=C_{2} .
$$

Proof. From (26) it follows that

$$
\lim _{k \rightarrow \infty} F\left(x_{k} / u_{k}\right)=\frac{1}{2}
$$

Since $F(x)$ is strictly decreasing and continuous it follows that the $\operatorname{limit}_{k \rightarrow \infty} \frac{u_{k}}{x_{k}}$ exists and equals $C_{1}=F^{-1}(1 / 2)$.

The limiting relation for $v_{k} / y_{k}$ is proved in exactly the same way.

In order to complete the proofs of Theorems 2.1 and 2.2 it remains to show the right tail estimates for $F_{k}$ and $G_{k}$.

Lemma 4.8 Let $1<\rho_{1}<\rho_{2}$ be defined as in Lemma 3.2. Then, for every $\rho$ that satisfies $1<\rho<\rho_{1}$ or $\rho>\rho_{2}$ there uniquely exists $F_{\rho}(x)$, that is the solution of the equation

$$
F_{\rho}(x / \rho)=\mathbf{E}\left(F_{\rho}\left(x V_{1}\right) \cdots F_{\rho}\left(x V_{m}\right)\right)
$$

and the normalization $L_{F_{\rho}}(1)=\frac{1}{2}$.

Furthermore, for $1<\rho<\rho_{1}$ we have $F_{\rho}(x)=1-d_{1} x^{\beta^{\prime}}+O\left(x^{\beta_{1}}\right)$ as $x \rightarrow 0+$ (for some constant $d_{1}>0$ ) and there exists $x_{1}>0$ and $C_{1}>0$ such that

$$
F_{\rho}(x) \leq e^{-C_{1} x^{\gamma}}
$$


for $x \geq x_{1}$, where $\gamma=(\log m) /(\log (m / \rho))$. Similarly, for $\rho>\rho_{2}$ we have $F_{\rho}=1-d_{2} x^{\beta^{\prime}}+O\left(x^{\beta_{2}}\right)$ as $x \rightarrow \infty$ (for some constant $d_{2}>0$ ) and there exists $x_{2}>0$ and $C_{2}>0$ such that

$$
F_{\rho}(x) \leq e^{-C_{2} x^{-\gamma^{\prime}}}
$$

for $x \geq x_{2}$, where $\gamma^{\prime}=(\log m) /(\log (\rho / m))$.

Note that $\rho_{2}>m$ so that $\gamma^{\prime}>0$.

Proof. First suppose that $1<\rho<\rho_{1}$ and let $\beta^{\prime}, \beta^{\prime \prime}$ denote the solutions of (11) that satisfy $0<\beta^{\prime}<\beta_{1}<\beta^{\prime \prime}$. Let $\mathcal{F}$ denote the set of non-negative, continuously decreasing functions $F(x)$, $x \geq 0$, that satisfy

$$
F(x)=1-x^{\beta^{\prime}}+O\left(x^{\beta_{1}}\right) \quad(x \rightarrow 0+) .
$$

(Note that all functions $F \in \mathcal{F}$ satisfy $0 \leq F(x) \leq 1$.) We observe that $\mathcal{F}$ endowed with the metric

$$
\delta(F, G)=\sup _{x \geq 0}\left|(F(x)-G(x)) x^{-\beta_{1}}\right|
$$

is a complete metric space. Furthermore the mapping

$$
S: F(x) \mapsto(S F)(x)=\mathbf{E}\left(F\left(\rho x V_{1}\right) \cdots F\left(\rho x V_{m}\right)\right)
$$

is a contraction on $\mathcal{F}$. First of all, if $F(x)$ is a non-negative, continuously decreasing function, then $(S F)(x)$ is a non-negative and continuously decreasing, too. Furthermore, if $F(x)$ satisfies (37) we also have (as $x \rightarrow 0+$ )

$$
\begin{aligned}
(S F)(x) & =1-m x^{\beta^{\prime}} \rho^{\beta^{\prime}} \mathbf{E}\left(V_{1}^{\beta^{\prime}}\right)+O\left(x^{\beta_{1}}\right) \\
& =1-x^{\beta^{\prime}} \rho^{\beta^{\prime}} \frac{(m(t+1)) !}{(t+1) !\left(\beta^{\prime}+t+1\right) \cdots\left(\beta^{\prime}+m(t+1)-1\right)}+O\left(x^{\beta_{1}}\right) \\
& =1-x^{\beta^{\prime}}+O\left(x^{\beta_{1}}\right) .
\end{aligned}
$$

Finally, suppose that $F, G \in \mathcal{F}$ with $\delta(F, G)=d$. Then $|F(x)-G(x)| \leq d x^{\beta_{1}}$ and we obtain

$$
\begin{aligned}
|(S F)(x)-(S G)(x)| & \leq m \mathbf{E}\left(\left|F\left(\rho x V_{1}\right)-G\left(\rho x V_{1}\right)\right|\right) \\
& \leq d m \rho^{\beta_{1}} x^{\beta_{1}} \mathbf{E}\left(V_{1}^{\beta_{1}}\right) \\
& =d \rho^{\beta_{1}} x^{\beta_{1}} \frac{(m(t+1)) !}{(t+1) !\left(\beta_{1}+t+1\right) \cdots\left(\beta_{1}+m(t+1)-1\right)}
\end{aligned}
$$

and consequently

$$
\delta((S F),(S G)) \leq \frac{(m(t+1)) ! \rho^{\beta_{1}}}{(t+1) !\left(\beta_{1}+t+1\right) \cdots\left(\beta_{1}+m(t+1)-1\right)} \cdot \delta(F, G) .
$$


Set

$$
g(\beta)=\frac{(m(t+1)) ! \rho^{\beta}}{(t+1) !(\beta+t+1) \cdots(\beta+m(t+1)-1)} .
$$

By Lemma 3.2 the equation $g(\beta)=1$ has only two solutions $\beta^{\prime}, \beta^{\prime \prime}>0$ and we have $g(\beta) \rightarrow \infty$ as $\beta \rightarrow \infty$ and if $\beta \rightarrow-t-1$. Since $\beta^{\prime}<\beta_{1}<\beta^{\prime \prime}$ we thus have $g\left(\beta_{1}\right)<1$. Therefore, the mapping $S: \mathcal{F} \rightarrow \mathcal{F}$ is a contraction.

Let $F_{\rho} \in \mathcal{F}$ denote the unique fixed point of $S$ that is granted by Banach's fixed point theorem. Our final goal is to show that $F_{\rho}$ satisfies (35). For this purpose set

$$
F_{0}(x)=\max \left\{1-x^{\beta^{\prime}}, 0\right\} \in \mathcal{F}
$$

and $F_{k+1}(x)=\left(S F_{k}\right)(x)$ for $k \geq 0$. By Banach's fixed point theorem this sequence of functions converges to $F_{\rho}$ and we have $\delta\left(F_{k}, F_{\rho}\right) \leq C$ for all $k \geq 0$ for some $C<\infty$. Consequently we know that

$$
F_{k}(x) \leq 1-x^{\beta^{\prime}}+C x^{\beta_{1}}=1-x^{\beta^{\prime}}\left(1-C x^{\beta_{1}-\beta^{\prime}}\right)
$$

for all $k \geq 0$ and $x \geq 0$. In particular there exists $x_{1}>0$ such that

$$
1-x^{\beta^{\prime}}+C x^{\beta_{1}}<1 \text { for } 0 \leq x \leq x_{1} .
$$

Next set

$$
\gamma=\frac{\log m}{\log (m / \rho)}
$$

and

$$
\eta=\min _{1 \leq \ell \leq m-1} \frac{\rho-\rho^{\frac{\log \ell}{\log m}}}{m-\ell}
$$

and choose $C_{1}>0$ such that

$$
1-x^{\beta^{\prime}}+C x^{\beta_{1}}<e^{-C_{1} x^{\gamma}} \text { for } \eta x_{1} \leq x \leq x_{1} .
$$

We now show inductively that

$$
F_{k}(x) \leq e^{-C_{1} x^{\gamma}} \quad\left(\text { for } x \geq x_{1}\right) .
$$

Obviously, (40) is satisfied for $k=0$. We assume that (40) is satisfied for some $k \geq 0$. By (38) and (39) we also have $F_{k}(x) \leq e^{-C_{1} x^{\gamma}}$ for $\eta x_{1} \leq x \leq x_{1}$. Our aim is to show that

$$
F_{k}\left(z_{1}\right) F_{k}\left(z_{2}\right) \cdots F_{k}\left(z_{m}\right) \leq e^{-C_{1} x^{\gamma}}
$$

for all $z_{1}, z_{2}, \ldots, z_{m} \geq 0$ with $z_{1}+z_{2}+\cdots+z_{m}=\rho x$. It is clear that (41) directly implies

$$
\begin{aligned}
F_{k+1}(x) & =\mathbf{E}\left(F_{k}\left(\rho x V_{1}\right) \cdots F_{k}\left(\rho x V_{m}\right)\right) \\
& \leq \mathbf{E}\left(e^{-C_{1} x^{\gamma}}\right)=e^{-C_{1} x^{\gamma}}
\end{aligned}
$$


for $x \geq x_{1}$ as proposed.

If $z_{i} \geq \eta x_{1}$ for all $i=1, \ldots, m$ then we directly get

$$
\begin{aligned}
F_{k}\left(z_{1}\right) F_{k}\left(z_{2}\right) \cdots F_{k}\left(z_{m}\right) & \leq e^{-C_{1}\left(z_{1}^{\gamma}+z_{2}^{\gamma}+\cdots+z_{m}^{\gamma}\right)} \\
& \leq e^{-C_{1} m\left(\left(z_{1}+z_{2}+\cdots+z_{m}\right) / m\right)^{\gamma}} \\
& =e^{-C_{1}\left(\left(z_{1}+z_{2}+\cdots+z_{m}\right) / \rho\right)^{\gamma}} \\
& =e^{-C_{1} x^{\gamma}}
\end{aligned}
$$

Next suppose that $z_{i} \geq \eta x_{1}$ for $1 \leq i \leq \ell$ and $z_{i}<\eta x_{1}$ for $\ell+1 \leq i \leq m$ for some $1 \leq \ell \leq m-1$. By symmetry this is no loss of generality. Furthermore, since $m \eta x_{1} \leq \rho x$ (for $x \geq x_{1}$ ) it cannot be the case that $z_{i}<\eta x_{1}$ for all $i$. Here we have

$$
\begin{aligned}
F_{k}\left(z_{1}\right) F_{k}\left(z_{2}\right) \cdots F_{k}\left(z_{m}\right) & \leq F_{k}\left(z_{1}\right) F_{k}\left(z_{2}\right) \cdots F_{k}\left(z_{\ell}\right) \\
& \leq e^{-C_{1}\left(z_{1}^{\gamma}+z_{2}^{\gamma}+\cdots+z_{\ell}^{\gamma}\right)} \\
& \leq e^{-C_{1} \ell\left(\left(z_{1}+z_{2}+\cdots+z_{\ell}\right) / \ell\right)^{\gamma}} \\
& =e^{-C_{1}(m / \ell)^{\gamma-1}\left(x-\left(z_{\ell+1}+\cdots+z_{m}\right) / \rho\right)^{\gamma}} \\
& \leq e^{-C_{1}(m / \ell)^{\gamma-1}\left(x-(m-\ell) \eta x_{1} / \rho\right)^{\gamma}} .
\end{aligned}
$$

Since

$$
\eta \frac{m-\ell}{\rho} \leq 1-\rho^{\frac{\log \ell}{\log m}-1}=1-\left(\frac{\ell}{m}\right)^{1-\gamma^{-1}}
$$

and $x_{1} \leq x$ it follows that

or

$$
\eta x_{1} \frac{m-\ell}{\rho} \leq x-x\left(\frac{\ell}{m}\right)^{1-\gamma^{-1}}
$$

$$
\left(\frac{m}{\ell}\right)^{\gamma-1}\left(x-(m-\ell) \frac{\eta x_{1}}{\rho}\right)^{\gamma} \geq x^{\gamma}
$$

which implies that

$$
F_{k}\left(z_{1}\right) F_{k}\left(z_{2}\right) \cdots F_{k}\left(z_{m}\right) \leq e^{-C_{1} x^{\gamma}}
$$

even in this remaining case. This completes the proof of (41). Note finally, that we can scale $F_{\rho}(x)$ properly so as to have $L_{F_{\rho}}(1)=\frac{1}{2}$.

If $\rho>\rho_{2}$ the proof is completely the same.

Lemma 4.9 Let $F_{k}(x)$ and $G_{k}(x)$ be defined as in Lemma 4.3 resp. Lemma 4.5, let $F(x)$ and $G(x)$ be given by Lemma 4.4 resp. Lemma 4.6, $\rho_{1}, \rho_{2}$ be defined as in Lemma 3.2, and $F_{\rho}(x)$ be given by Lemma 4.8.

If $1<\rho<\rho_{1}$ then

$$
\begin{aligned}
& \frac{1}{u_{k}} L_{F_{k}}\left(\frac{u}{u_{k}}\right) \leq L_{F}(u) \leq L_{F_{\rho}}(u) \quad(\text { for } u<1,) \\
& \frac{1}{u_{k}} L_{F_{k}}\left(\frac{u}{u_{k}}\right) \geq L_{F}(u) \geq L_{F_{\rho}}(u) \quad(\text { for } u>1 .)
\end{aligned}
$$


If $\rho>\rho_{2}$ then

$$
\begin{aligned}
& \frac{1}{v_{k}} L_{G_{k}}\left(\frac{u}{v_{k}}\right) \geq L_{G}(u) \leq L_{F_{\rho}}(u) \quad(\text { for } 0<u<1,) \\
& \frac{1}{v_{k}} L_{G_{k}}\left(\frac{u}{v_{k}}\right) \leq L_{G}(u) \geq L_{F_{\rho}}(u) \quad(\text { for } u>1 .)
\end{aligned}
$$

Proof. First suppose that $1<\rho<\rho_{1}$. We set

$$
\tilde{F}_{j}(x)=F_{j}\left(x u_{k}\right) \quad \text { and } \quad \tilde{G}_{j}(x)=F_{\rho}\left(x \rho^{k-j}\right) .
$$

Then $\tilde{G}_{0}(x)-\tilde{F}_{0}(x)$ has exactly one "zero" which implies that all derivatives of the Laplace transform have the same property. Furthermore, $L_{\tilde{F}_{k}}(1)=L_{\tilde{G}_{k}}(1)$. We are, thus, in the same situation as in the proof of Lemma 4.3. Consequently we obtain

$$
\begin{aligned}
& \frac{1}{u_{k}} L_{F_{k}}\left(\frac{u}{u_{k}}\right) \leq L_{F_{\rho}}(u) \quad(\text { for } u<1 \text { and }) \\
& \frac{1}{u_{k}} L_{F_{k}}\left(\frac{u}{u_{k}}\right) \geq L_{F_{\rho}}(u) \quad(\text { for } u>1 .)
\end{aligned}
$$

This is true for all $k$. Hence we also get $L_{F}(u) \leq L_{F_{\rho}}(u)$ for $u<1$ and $L_{F}(u) \geq L_{F_{\rho}}(u)$ for $u>1$.

The case $\rho>\rho_{2}$ can be treated in completely the same way.

Lemma 4.10 Let $F_{k}(x)$ and $G_{k}(x)$ be as above. Then there exist constants $C_{1}, C_{2}>0, \gamma_{1}, \gamma_{2}>$ 1 , and $\beta_{1}, \beta_{2}>0$ with

$$
\begin{aligned}
F_{k}(x) & =O\left(e^{-C_{1}\left(x / u_{k}\right)^{\gamma_{1}}}\right) \quad\left(\text { for } x \geq u_{k}\right), \\
1-F_{k}(x) & =O\left(\left(x / u_{k}\right)^{\beta_{1}}\right) \quad\left(\text { for } x \leq u_{k}\right), \\
G_{k}(x) & =O\left(e^{-C_{2}\left(x / v_{k}\right)^{-\gamma_{2}}}\right) \quad\left(\text { for } x \leq v_{k}\right), \\
1-G_{k}(x) & =O\left(\left(x / v_{k}\right)^{-\beta_{2}}\right) \quad\left(\text { for } x \geq v_{k}\right)
\end{aligned}
$$

Proof. By $1-F_{\rho}(x) \sim x^{\beta^{\prime}}$ and (35) it follows that

$$
L_{1-F_{\rho}}(u)=O\left(u^{-1-\beta^{\prime}}\right) \quad(u \rightarrow \infty)
$$

and

$$
L_{F_{\rho}}(u)=O\left(e^{-C_{2}(-u)^{\gamma /(\gamma-1)}}\right) \quad(u \rightarrow-\infty) .
$$


Now assume that $0<x<\beta^{\prime}$ and set $u=\beta^{\prime} / x$ (for which we have $u>1$ ). With the help of Lemma 4.9 we, thus, obtain

$$
\begin{aligned}
L_{1-F_{\rho}}(u) & \geq \frac{1}{u_{k}} L_{1-F_{k}}\left(\frac{u}{u_{k}}\right) \\
& =\int_{0}^{\infty}\left(1-F_{k}\left(t u_{k}\right)\right) e^{-t u} d t \\
& \geq\left(1-F_{k}\left(x u_{k}\right)\right) \int_{x}^{\infty} e^{-t u} d t \\
& =\left(1-F_{k}\left(x u_{k}\right)\right) \frac{e^{-u x}}{u}
\end{aligned}
$$

and consequently

$$
1-F_{k}\left(x u_{k}\right)=O\left(e^{u x} u^{-\beta^{\prime}}\right)=O\left(x^{\beta^{\prime}}\right) .
$$

Similarly we obtain for $u=-c x^{\gamma-1}<0$ (with a properly chosen constant $c>0$ )

$$
\begin{aligned}
L_{F_{\rho}}(u) & \geq \frac{1}{u_{k}} L_{F_{k}}\left(\frac{u}{u_{k}}\right) \\
& =\int_{0}^{\infty} F_{k}\left(t u_{k}\right) e^{-t u} d t \\
& \geq F_{k}\left(x u_{k}\right) \int_{0}^{x} e^{-t u} d t \\
& \geq F_{k}\left(x u_{k}\right) \frac{e^{-u x}}{-u}
\end{aligned}
$$

and finally

$$
F_{k}\left(x u_{k}\right)=O\left(u e^{u x+C_{1} x^{\gamma}}\right)=O\left(e^{-C_{2} x^{\gamma /(\gamma-1)}}\right) .
$$

The bounds for $G_{k}(x)$ follow along similar lines.

By combining the preceding results we have completed the proofs Theorem 2.1 and Theorem 2.2.

\subsection{Height and Saturation Level of $m$-Ary Search Trees}

The proof of Theorem 2.3 is very similar to that of Theorem 2.1 and Theorem 2.2. A major tool is again an intersection property stated in Lemma 4.12.

Lemma 4.11 Let $H_{n}^{(m, t)}$ denote the height and $\bar{H}_{n}^{(m, t)}$ the saturation level of fringe balanced $m$-ary search trees. Then the generating functions

$$
y_{k}(x)=\sum_{n \geq 0} \operatorname{Pr}\left\{H_{n}^{(m, t)} \leq k\right\} \cdot x^{n} \quad \text { and } \quad \bar{y}_{k}(x)=\sum_{n \geq 0} \operatorname{Pr}\left\{\bar{H}_{n}^{(m, t)} \geq k\right\} \cdot x^{n}
$$


satisfy the recurrence relation

$$
\begin{aligned}
& y_{k+1}^{(m(t+1)-1)}(x)=\frac{(m(t+1)-1) !}{(t !)^{m}}\left(y_{k}^{(t)}(x)\right)^{m}, \\
& \bar{y}_{k+1}^{(m(t+1)-1)}(x)=\frac{(m(t+1)-1) !}{(t !)^{m}}\left(\bar{y}_{k}^{(t)}(x)\right)^{m}
\end{aligned}
$$

with initial conditions

$$
y_{0}(x)=1, y_{k}(0)=y_{k}^{\prime}(0)=\cdots=y_{k}^{(m-1)}(0)=1
$$

and

$$
\bar{y}_{0}(x)=\frac{x}{1-x}, y_{k}(0)=y_{k}^{\prime}(0)=\cdots=y_{k}^{(m-1)}(0)=0
$$

Proof. The recurrence (42) is just a restatement of (4).

Lemma 4.12 Let $y_{0}(x), y_{1}(x), z_{0}(x), z_{1}(x)$ be non-negative functions for $x \geq 0$ such that the difference $y_{0}^{(t)}(x)-z_{0}^{(t)}(x)$ has exactly one positive zero, that

$$
\begin{aligned}
& y_{1}^{(m(t+1)-1)}(x)=\frac{(m(t+1)-1) !}{(t !)^{m}}\left(y_{0}^{(t)}(x)\right)^{m}, \\
& z_{1}^{(m(t+1)-1)}(x)=\frac{(m(t+1)-1) !}{(t !)^{m}}\left(z_{0}^{(t)}(x)\right)^{m},
\end{aligned}
$$

and that $y_{1}^{(j)}(0)-z_{1}^{(j)}(0)>0$ for $j=0,1, \ldots, m(t+1)-1$. Then, the differences $y_{1}^{(j)}(x)-z_{1}^{(j)}(x)$, $j=0,1, \ldots, m(t+1)-1$, have at most one positive zero.

Proof. The proof is completely similar to the proof Lemma 4.2 (see also [13]).

Lemma 4.13 Let $c_{k}$ and $d_{k}$ be defined by the relations

$$
\frac{1}{c_{k}} y_{k}\left(1-\frac{1}{c_{k}}\right)=\frac{1}{2} \quad \text { and } \quad \frac{1}{d_{k}} \bar{y}_{k}\left(1-\frac{1}{d_{k}}\right)=\frac{1}{2} .
$$

Then we have that

$$
\begin{aligned}
& \frac{1}{c_{k}} y_{k}\left(1-\frac{u}{c_{k}}\right)<\frac{1}{c_{k+1}} y_{k+1}\left(1-\frac{u}{c_{k+1}}\right) \quad(\text { for } u<1,) \\
& \frac{1}{c_{k}} y_{k}\left(1-\frac{u}{c_{k}}\right)>\frac{1}{c_{k+1}} y_{k+1}\left(1-\frac{u}{c_{k+1}}\right) \quad(\text { for } u>1) .
\end{aligned}
$$

and similar estimates follow for $\bar{y}_{k}(x)$.

Proof. The proof is almost the same as that of Lemma 4.3. 
Lemma 4.14 Let $c_{k}$ be as defined in Lemma 4.13. Then the limit

$$
L_{1}(u)=\lim _{k \rightarrow \infty} \frac{1}{c_{k}} y_{k}\left(1-\frac{u}{c_{k}}\right)
$$

exists for each real $u>0$ and $L_{1}(u)$ satisfies the differential equation

$$
L_{1}^{(m(t+1)-1)}(u)=(-1)^{m-1} \frac{(m(t+1)-1) !}{(t !)^{m}}\left(\frac{1}{\rho_{1}^{t+1}} L_{1}^{(t)}\left(u / \rho_{1}\right)\right)^{m},
$$

where

$$
\rho_{1}=\lim _{k \rightarrow \infty} \frac{c_{k+1}}{c_{k}},
$$

that exists, too. Hence, $L_{1}(u)$ is the same function as in Lemma 4.3 and is the Laplace transform of a function $F(x)$, for which $1-F(x)$ is a distribution function.

We also have

$$
\operatorname{Pr}\left\{H_{n}^{(t)} \leq k\right\}=F\left(n / c_{k}\right)+o(1)
$$

uniformly for $k \geq 0$, as $n \rightarrow \infty$.

Furthermore, there exist constants $C_{1}>0, \gamma_{1}>1$, and $\beta_{1}>0$ such that

$$
\begin{aligned}
& \operatorname{Pr}\left\{H_{n}^{(t)} \leq k\right\}=O\left(e^{-C_{1}\left(n / c_{k}\right)^{\gamma_{1}}}\right) \quad\left(\text { for } n \geq c_{k}\right), \\
& \operatorname{Pr}\left\{H_{n}^{(t)}>k\right\}=O\left(\left(n / c_{k}\right)^{\beta_{1}}\right) \quad\left(\text { for } n \leq c_{k}\right) .
\end{aligned}
$$

Proof. By Lemma 4.13 it is clear that the limit $L_{1}(u)$ exists and the convergence is also uniform on any interval of the form $\left[u^{\prime}, u^{\prime \prime}\right] \subseteq(0, \infty)$.

We set $F_{k}(x)=\operatorname{Pr}\left\{H_{x}^{(t)} \leq k\right\}$ if $x \geq 0$ is an integer and by linear interpolation for nonintegral $x \geq 0$. Then $1-F_{k}(x)$ is a distribution function and we have (for every fixed $u \geq 0$ and as $k \rightarrow \infty)$

$$
\begin{aligned}
\frac{1}{c_{k}} y_{k}\left(1-\frac{u}{c_{k}}\right) & =\sum_{n \geq 0} \operatorname{Pr}\left\{H_{n}^{(t)} \leq k\right\}\left(1-u / c_{k}\right)^{n} \frac{1}{c_{k}} \\
& =\int_{0}^{\infty} F_{k}\left(y c_{k}\right) e^{-u y} d y+o(1) .
\end{aligned}
$$

Hence, we can argue as in the proof of Lemma 4.4 and it follows that there exists $F(x)$ (that has Laplace transform $\left.L_{1}(u)\right)$ with $F_{k}\left(x c_{k}\right)=F(x)+o(1)$ for all continuity points of $F$.

Next, (42) can be rewritten to

$$
\frac{1}{c_{k+1}^{t+1}} y_{k+1}^{(m(t+1)-1)}\left(1-u / c_{k+1}\right)=(-1)^{m-1} \frac{(m(t+1)-1) !}{(t !)^{m}}\left(\frac{1}{\kappa_{k}^{t+1}} \frac{1}{c_{k}^{t+1}} y_{k}^{(t)}\left(1-u /\left(\kappa_{k} c_{k}\right)\right)\right)^{m}
$$


which leads to

$$
L_{1}^{(m(t+1)-1)}(u)=(-1)^{m-1} \frac{(m(t+1)-1) !}{(t !)^{m}} \lim _{k \rightarrow \infty}\left(\frac{1}{\kappa_{k}^{t+1}} L_{1}^{(t)}\left(u / \kappa_{k}\right)\right)^{m},
$$

where $\kappa_{k}=c_{k+1} / c_{k}$, and as in the proof of Lemma 4.4, it follows that the limit $\rho^{\prime}=\lim _{k \rightarrow \infty} \kappa_{k}=$ $\lim _{k \rightarrow \infty} c_{k+1} / c_{k}$ exists.

Since we know that $H_{n}^{(m, t)} / \log n \rightarrow 1 /\left(\log \rho_{1}\right)$ (almost surely as $\left.n \rightarrow \infty\right), \rho^{\prime}$ has to be equal to $\rho_{1}$. Hence, $L_{1}(u)$ and $F(x)$ are (up to scaling) the same functions as those in Lemma 4.3.

Next, the tail estimates (47) and (48) follow in the same way in in the proofs of Lemma 4.10 (see also [13]). The only difference is that the Laplace integral has to be replaced by the power series $y_{k}(x)$.

Putting all these things together we have shown that there exists a sequence $\varepsilon_{k} \rightarrow 0$ with

$$
\left|\operatorname{Pr}\left\{H_{n}^{(m, t)} \leq k\right\}-F\left(n / c_{k}\right)\right| \leq \varepsilon_{k}
$$

uniformly for all $n \geq 0$. Indeed, this is not exactly what we want to show. However, by combining this estimate with the tail estimates for $\operatorname{Pr}\left\{H_{n}^{(t)} \leq k\right\}$ and $F(x)$ it is easy to see that (46) follows, too.

The corresponding property for the saturation level $\bar{H}_{n}^{(m, t)}$ follows in analogous manner to that of $H_{n}^{(m, t)}$. Theorem 2.3 is now an immediate consequence of Lemma 4.14.

\section{The Intersection Property}

In this final section we consider discrete branching random walks $Z_{k}$ defined by the point process

$$
Z=\sum_{j=1}^{N} \delta_{X_{j}}
$$

where we assume that $\mathbf{E} N>1$ and $\operatorname{Pr}\{N<\infty\}=1$.

It is well known that if $G_{k}(x)=\operatorname{Pr}\left\{R_{k} \leq x\right\}$ is the distribution function of the rightmost particle at level $k$ then $G_{k+1}(x)$ is given by

$$
G_{k+1}(x)=\mathbf{E}\left(\prod_{j=1}^{N} G_{k}\left(x-X_{j}\right)\right) .
$$

This motivates us to define the transform $T$ :

$$
(T G)(x)=\mathbf{E}\left(\prod_{j=1}^{N} G\left(x-X_{j}\right)\right) .
$$

In what follows we assume that $T$ satisfies the intersection property: 
Suppose that $F(x)$ and $G(x)$ are continuous distribution functions such that the difference $F(x)-G(x)$ has exactly one zero. Then the difference $(T F)(x)-(T G)(x)$ has at most one zero.

It is worth mentioning that there are several examples where the intersection property applies. For example, if $N \geq 2$ is constant and if the $X_{j}, 1 \leq j \leq N$ are iid with log-concave density then the intersection property holds, compare with Bachmann [2]. Note further that $F(x) \leq G(x)$ implies $(T F)(x) \leq(T G)(x)$ (resp. no zero implies no zero) and that $T$ is sign preserving in the sense that if $F(x)-G(x)$ and $(T F)(x)-(T G)(x)$ have exactly one zero then $F(x)-G(x)>0$ as $x \rightarrow \infty$ if and only if $(T F)(x)-(T G)(x)>0$ as $x \rightarrow \infty$.

We will also make the following general assumption on $Z$. We assume that

$$
\bar{m}(\beta)=\mathbf{E}\left(\sum_{j=1}^{N} e^{-\beta X_{j}}\right)
$$

exists in a neighborhood of $\beta=0$ and that the equation

$$
\frac{\log \bar{m}(\beta)}{\beta}=\frac{\bar{m}^{\prime}(\beta)}{\bar{m}(\beta)}
$$

has in this neighborhood exactly two solutions $\beta_{1}$ and $\beta_{2}$ which are the same as in Lemma 3.2 in the particular case of the fragmentation problem. Without loss of generality we can assume that the velocities $c_{1}=-\left(\log \bar{m}\left(\beta_{1}\right)\right) / \beta_{1}$ and $c_{2}=-\left(\log \bar{m}\left(\beta_{2}\right)\right) / \beta_{2}$ satisfy $0<c_{1}<c_{2}$.

The following theorem provides convergence to a travelling wave $w(x)$. It has to be compared with Theorem 2.2, where the intersection property is not put ahead.

Theorem 5.1 Let $Z_{k}$ be a branching random walk as described above, in particular the intersection property holds. Let $L_{k}$ resp. $R_{k}$ the position of the leftmost resp. the rightmost particle at level $k$. Then there exist functions $w_{1}(x)$ and $w_{2}(x)$ such that

$$
\operatorname{Pr}\left\{L_{k}>x\right\}=w_{1}\left(x-m_{1}(k)\right)+o(1) \quad \text { and } \quad \operatorname{Pr}\left\{R_{k} \leq x\right\}=w_{2}\left(x-m_{2}(k)\right)+o(1),
$$

where $m_{1}(k)$ and $m_{2}(k)$ are defined by $\operatorname{Pr}\left\{L_{k} \leq m_{1}(k)\right\}=\operatorname{Pr}\left\{R_{k} \leq m_{2}(k)\right\}=1 / 2$. They are asymptotically given by

$$
m_{1}(k)=k c_{1}+o(k) \quad \text { and } \quad m_{2}(k)=k c_{2}+o(k) .
$$

Moreover, the limits $c_{1}=\lim _{k \rightarrow \infty}\left(m_{1}(k+1)-m_{1}(k)\right)$ and $c_{2}=\lim _{k \rightarrow \infty}\left(m_{2}(k+1)-m_{2}(k)\right)$ exist and $w_{1}(x), w_{2}(x)$ are (up to shifts) the unique solutions of the equations

$$
w_{1}(x)=\mathbf{E}\left(\prod_{j=1}^{N} w_{1}\left(x+c_{1}-X_{j}\right)\right) \quad \text { and } \quad w_{2}(x)=\mathbf{E}\left(\prod_{j=1}^{N} w_{2}\left(x+c_{2}-X_{j}\right)\right) .
$$


Furthermore suppose that $Z$ satisfies the property that there exist constants $\bar{n}>0$ and $\gamma^{\prime}>0$ such that a.s.

$$
N \leq \bar{n} \quad \text { and } \quad \sum_{j=1}^{N} e^{-\gamma X_{j}} \geq 1 \quad\left(0 \leq \gamma \leq \gamma^{\prime}\right)
$$

Then there exist $C>0$ and $\eta>0$ with

$$
\operatorname{Pr}\left\{\left|L_{k}-m_{1}(k)\right|>x\right\} \leq C e^{-\eta x} \quad \text { and } \quad \operatorname{Pr}\left\{\left|R_{k}-m_{2}(k)\right|>x\right\} \leq C e^{-\eta x} .
$$

In particular we have, as $k \rightarrow \infty$, that

$$
\operatorname{Var} L_{k}=O(1) \quad \text { and } \quad \operatorname{Var} R_{k}=O(1) .
$$

The branching random walk framework allows to get a martingale representation for $w(x)$. It is detailed in the following theorem.

Theorem 5.2 Let $w_{1}$ and $w_{2}$ be as given in Theorem 5.1. Then for every real $x$

$$
M_{n}(x)=\prod_{|u|=n} w_{1}\left(x+n c_{1}-X_{u}\right)
$$

is a multiplicative martingale with expectation $w_{1}(x)$, it converges a.s. and in $L^{1}$ to a nondegenerate limit which can be written as

$$
M(x)=\exp (-Z(x))
$$

Moreover,

$$
w_{1}(x)=\mathbf{E}\left(\exp \left\{-e^{\beta_{1} x} Z(0)\right\}\right)
$$

which allows to represent any solution $\Phi$ of equation (8) as a Laplace transform of a limit of martingales, namely

$$
\Phi(y)=\mathbf{E}\left(e^{-y Z(0)}\right) .
$$

Theorem 5.2 relies on arguments contained in [15]. As noticed in [15], the two representations: in (15) the Laplace transform of $W^{\prime}\left(\beta_{1}\right)$ (an additive martingale limit) and in (49) the Laplace transform of $Z(0)$ (a multiplicative martingale limit) coincide thanks to the tail distribution of $\Phi$ given in (9).

For the sake of shortness we do not give a proof of Theorem 5.2 but we indicate the main steps of the proof of Theorem 5.1. It is split into several lemmas.

Lemma 5.3 Suppose that the intersection property holds. Let $F_{0}(x)$ be defined by

$$
F_{0}(x)= \begin{cases}1 & \text { for } x<0 \\ 0 & \text { for } x \geq 0\end{cases}
$$


and recursively by $F_{k+1}=T F_{k}$ for $k \geq 0$. Furthermore, let $m_{1}(k)>0$ be defined by

$$
F_{k}\left(m_{1}(k)\right)=\frac{1}{2}
$$

Then we have that

$$
\begin{array}{ll}
F_{k+1}\left(x+m_{1}(k+1)\right) \leq F_{k}\left(x+m_{1}(k)\right) & (\text { for } x<0,) \\
F_{k+1}\left(x+m_{1}(k+1)\right) \geq F_{k}\left(x+m_{1}(k)\right) & (\text { for } x>0 .)
\end{array}
$$

Consequently, the limit

$$
w_{1}(x)=\lim _{k \rightarrow \infty} F_{k}\left(x+m_{1}(k)\right)
$$

exists and is uniform for all $x$. Furthermore

$$
c_{1}=\lim _{k \rightarrow \infty}\left(m_{1}(k+1)-m_{1}(k)\right)
$$

and $w_{1}(x)$ satisfies the functional equation

$$
w_{1}(x)=\mathbf{E}\left(\prod_{j=1}^{N} w_{1}\left(x+c_{1}-X_{j}\right)\right) .
$$

Proof. First of all, by the existence of $\bar{m}(\beta)$ in a neighbourhood of $\beta=0$ there are proper tail estimates and it easily follows that $\lim _{x \rightarrow \infty} F_{k}(x)=0$ and $\lim _{x \rightarrow-\infty} F_{k}(x)=1$ (for all $k \geq 1$ ). Furthermore, $F_{k}(x)$ is continuous and strictly decreasing. Hence, $m_{1}(k)$ is uniquely defined.

Now fix some $k \geq 1$ and define $\tilde{F}_{j}(x)=F_{j}\left(x+m_{1}(k)\right)$ and $\tilde{G}_{j}(x)=F_{j+1}\left(x+m_{1}(k+1)\right.$ ) (for $j=0,1, \ldots, k)$. By definition we have $\tilde{F}_{k}(0)=\tilde{G}_{k}(0)=1 / 2$. Furthermore, $\tilde{G}_{0}(x)-\tilde{F}_{0}(x)$ has at most one "zero". Hence, by the intersection property it follows that all differences $\tilde{G}_{j}(x)-\tilde{F}_{j}(x)$, $j=0,1, \ldots, k$, have exactly one zero and $\tilde{G}_{j}(x)-\tilde{F}_{j}(x)>0$ as $x \rightarrow \infty$. In particular we obtain (50) and (51).

Consequently, the limit (52) exists uniformly for all $x$ and we also obtain

$$
\lim _{k \rightarrow \infty} w_{1}\left(x+m_{1}(k+1)-m_{1}(k)\right)=\left(T w_{1}\right)(x)
$$

for all $x$. Hence, the limit (53) exists and has to be equal to $c_{1}$ (due to general properties of branching random walks, compare with Biggins [4]). Finally, we also obtain (54).

Note that $F_{k}(x)=\operatorname{Pr}\left\{L_{k}>x\right\}$ and that there is a completely analogous lemma for $G_{k}(x)=\operatorname{Pr}\left\{R_{k} \leq x\right\}$ that we do not state explicitly.

Lemma 5.4 Let $0<c_{1}=-\left(\log \bar{m}\left(\beta_{1}\right)\right) / \beta_{1}<c_{2}=-\left(\log \bar{m}\left(\beta_{2}\right)\right) / \beta_{2}$ be the velocities from above. Then for $c$ with $0<c<c_{1}$ resp. for $c>c_{2}$ the equation

$$
\beta c=-\log \bar{m}(\beta)
$$


has exactly two solutions $\beta^{\prime}, \beta^{\prime \prime}$ that satisfy $\beta^{\prime}<\beta_{1}<\beta^{\prime \prime}$ resp. $\beta^{\prime}<\beta_{2}<\beta^{\prime \prime}$. If $c_{1}<c<c_{2}$ there are no solutions, and if $c=c_{1}$ (resp. $c=c_{2}$ ) then there is exactly one solution $\beta=\beta_{1}$ (resp. $\beta=\beta_{2}$ ).

Proof. The proof is of the same style as that of Lemma 3.2.

Lemma 5.5 For every $c$ that satisfies $0<c<c_{1}$ or $c>c_{2}$ there uniquely exists $w_{c}(x)$, that is the solution of the equation

$$
w_{c}(x)=\mathbf{E}\left(\prod_{j=1}^{N} w_{c}\left(x+c-X_{j}\right)\right)
$$

and satisfies the normalization $w_{c}(0)=\frac{1}{2}$.

Furthermore suppose that $Z$ satisfies the property that there exist $\bar{n}>0$ and $\gamma^{\prime}>0$ such that a.s.

$$
N \leq \bar{n} \quad \text { and } \quad \sum_{j=1}^{N} e^{-\gamma X_{j}} \geq 1 \quad\left(0 \leq \gamma \leq \gamma^{\prime}\right) .
$$

Then for $0<c<c_{1}$ we have $w_{c}(x)=1-d_{1} e^{\beta^{\prime} x}+O\left(e^{\beta_{1} x}\right)$ as $x \rightarrow-\infty$ and there exist $x_{1}$ and $C_{1}>0, \eta_{1}>0$ such that

$$
w_{c}(x) \leq \exp \left(-C_{1} e^{\eta_{1} x}\right)
$$

for $x \geq x_{1}$. Similarly, for $c>c_{2}$ we have $w_{c}(x)=1-d_{2} e^{\beta^{\prime} x}+O\left(e^{\beta_{2} x}\right)$ as $x \rightarrow \infty$ and there exist $x_{2}$ and $C_{2}>0, \eta_{2}>0$ such that

$$
w_{c}(x) \leq \exp \left(-C_{2} e^{-\eta_{2} x}\right)
$$

for $x \leq x_{2}$.

Proof. The proof is very close to that of Lemma 4.8; and we only consider the case $0<c<c_{1}$.

We consider the set $\mathcal{F}$ of functions $w(x)$ that are continuous and strictly decreasing and satisfy $w(x)=1-e^{\beta^{\prime} x}+O\left(e^{\beta_{1} x}\right)$ as $x \rightarrow-\infty$ and $\lim _{x \rightarrow \infty} w(x)=0$. The existence of $w_{c}$ follows from the Banach's fixed point theorem.

The proof of (56) is more involved. Without loss of generality we can assume that $\gamma<\beta^{\prime}$. Then we have $\bar{m}(\gamma)>e^{-\gamma c}$ which ensures that a.s. there exists $j$ with $X_{j} \leq c+\log \bar{n} / \gamma$. Next we fix $\eta>\gamma$ and $D>\log \bar{n} / \gamma$ such that

$$
e^{\eta c} J^{1-\eta / \gamma}\left(1-(\bar{n}-J) e^{-\gamma(c+D)}\right)^{\eta / \gamma} \geq 1
$$

for $J=1,2, \ldots, \bar{n}$ (which is surely possible by continuity). 
The main step of the proof is to observe that an estimate of the form $w(x) \leq \exp \left(-C e^{\eta x}\right)$ for $x \geq x_{1}-D$ implies that

$$
\tilde{w}(x)=\mathbf{E}\left(\prod_{j=1}^{N} w\left(x+c-X_{j}\right)\right) \leq \exp \left(-C e^{\eta x}\right)
$$

for $x \geq x_{1}$, compare with the proof of Lemma 4.8.

By assumption we have a.s. $1 \leq J=|S| \leq \bar{n}$, where

$$
S=\left\{j \geq 1: X_{j} \leq c+D\right\} \text {. }
$$

Consequently

$$
\sum_{j \in S} e^{-\gamma X_{j}} \geq 1-(\bar{n}-J) e^{-\gamma(c+D)}
$$

If $x \geq x_{1}$ and $X_{j} \leq c+D$ then $x+c-X_{j} \geq x_{1}-D$. Hence, we get a.s.

$$
\begin{aligned}
\prod_{j=1}^{N} w\left(x+c-X_{j}\right) & \leq \prod_{j \in S} \exp \left(-C e^{\eta(x+c)} e^{-\eta X_{j}}\right) \\
& =\exp \left(-C e^{\eta(x+c)} \sum_{j \in S}\left(e^{-\gamma X_{j}}\right)^{\eta / \gamma}\right) \\
& \leq \exp \left(-C e^{\eta(x+c)} J^{1-\eta / \gamma}\left(\sum_{j \in S} e^{-\gamma X_{j}}\right)^{\eta / \gamma}\right) \\
& \leq \exp \left(-C e^{\eta(x+c)} J^{1-\eta / \gamma}\left(1-(\bar{n}-J) e^{-\gamma(c+D)}\right)^{\eta / \gamma}\right) \\
& \leq \exp \left(-C e^{\eta x}\right)
\end{aligned}
$$

and thus $\tilde{w}(x) \leq \exp \left(-C e^{\eta x}\right)$ for $x \geq x_{1}$.

Lemma 5.6 Suppose that the intersection property holds. Let $F_{k}(x)$ be defined as in Lemma 5.3 and $w_{c}$ be given as in Lemma 5.5. If $0<c<c_{1}$ then

$$
\begin{aligned}
& F_{k}\left(x+m_{1}(k)\right) \geq w_{1}(x) \geq w_{c}(x) \quad(\text { for } x<0,) \\
& F_{k}\left(x+m_{1}(k)\right) \leq w_{1}(x) \leq w_{c}(x) \quad(\text { for } x>0 .)
\end{aligned}
$$

Proof. The proof is almost the same as that of Lemma 4.9.

By combining Lemma 5.3-5.6 (with analogue results for $w_{2}(x)$ ) one immediately derives Theorem 5.1.

Acknowledgement. The authors are thankful to the referees for their careful reading of the first version of this paper and for their valuable remarks to improve the presentation of the paper. 


\section{References}

[1] J.-F. Marckert B. Chauvin, T. Klein and A. Rouault. Martingales and profile of binary search trees. Electronic Journal In. Prob., 10:420-435, 2005.

[2] M. Bachmann. Limit theorems for the minimal position in a branching random walk with independent logconcave displacements. Adv. Appl. Prob., 32:159-176, 2000.

[3] J. Bertoin. Self-similar fragmentations. Ann. Inst. H. Poincaré Probab. Statist., 38(3):319$340,2002$.

[4] J. D. Biggins. Chernoff's theorem in the branching random walk. J. Appl. Prob., 14:630-636, 1977.

[5] J. D. Biggins and A. E. Kyprianou. Seneta-Heyde norming in the branching random walk. Ann. Probab., 25:337-360, 1997.

[6] J. D. Biggins and A. E. Kyprianou. Measure change in multitype branching. Adv. Appl. Prob., 36:544-581, 2004.

[7] J. D. Biggins and A. E. Kyprianou. The smoothing transform: the boundary case. Electronic Journal In. Prob., 10:609-631, 2005.

[8] M. Bramson. Convergence of solutions of the kolmogorov equation to travelling waves. Memoirs of the AMS, 44(285):1-190, 1983.

[9] B. Chauvin and A. Rouault. Connecting Yule process, bisection and binary search tree via martingales. JIRSS, 3:88-116, 2004.

[10] L. Devroye. A note on the height of binary search trees. J. Assoc. Comput. Mach., 33:489498, 1986.

[11] L. Devroye. On the height of random $m$-ary search trees. Random Struc. Algorithms, 1:191-203, 1990.

[12] L. Devroye. Universal limit laws for depths in random trees. SIAM J. Comput., 28(2):409432 (electronic), 1999.

[13] M. Drmota. An analytic approach to the height of binary search trees II. J. Assoc. Comput. Mach., 50:333-374, 2003.

[14] R. Durrett and T. Liggett. Fixed points of the smoothing transformation. Z. Wahrsch. Verw. Gebiete, 64(3):275-301, 1983.

[15] A. E. Kyprianou. Slow variation and uniqueness of solutions to the functional equation in the branching random walk. J. Appl. Prob., 35:795-801, 1998. 
[16] Q. Liu. Fixed points of a generalized smoothing transformation and applications to the branching random walk. Adv. Appl. Prob., 30:85-112, 1998.

[17] H. M. Mahmoud. Evolution of random search trees. Wiley-Interscience Series in Discrete Mathematics and Optimization. John Wiley \& Sons Inc., New York, 1992.

[18] S. N. Majumdar and P. L. Krapivsky. Traveling waves, front selection, and exact nontrivial exponents in a random fragmentation problem. Phys. Rev. Lett., 85:5492-5495, 2000.

[19] S. N. Majumdar and P. L. Krapivsky. Extreme value statistics and traveling fronts: various applications. Phys. A, 318(1-2):161-170, 2003.

[20] C. McDiarmid. Minimal positions in a branching random walk. Ann. Appl. Probab., 5(1):128-139, 1995.

[21] B. Pittel. On growing random binary trees. J. Math. Anal. Appl., 103:461-480, 1984.

[22] B. Reed. The height of a random binary search tree. J. Assoc. Comput. Mach., 50:306-332, 2003.

[23] J. M. Robson. The height of binary search trees. Austral. Comput. J., 11:151-153, 1979. 